TID-8200(22nd Rev.)

\title{
MASTER
}

\section{NUCLEAR REACTORS}

\section{BUILT,}

BEING BUILT,

or PLANNED

in the UNITED STATES as of

June 30,1970

\section{Prepared by Office of the Assistant General Manager for Reactors}

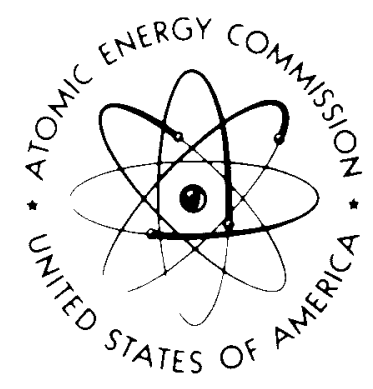

- United States Atomic Energy Commission Division of Technical Information 


\section{DISCLAIMER}

This report was prepared as an account of work sponsored by an agency of the United States Government. Neither the United States Government nor any agency Thereof, nor any of their employees, makes any warranty, express or implied, or assumes any legal liability or responsibility for the accuracy, completeness, or usefulness of any information, apparatus, product, or process disclosed, or represents that its use would not infringe privately owned rights. Reference herein to any specific commercial product, process, or service by trade name, trademark, manufacturer, or otherwise does not necessarily constitute or imply its endorsement, recommendation, or favoring by the United States Government or any agency thereof. The views and opinions of authors expressed herein do not necessarily state or reflect those of the United States Government or any agency thereof. 


\section{DISCLAIMER}

Portions of this document may be illegible in electronic image products. Images are produced from the best available original document. 
Price: $\$ 3.00$

Copies of this report may be purchased from

National Technical Information Service

U. S. Department of Commerce

Springfield, Virginia 22151

Requests should include the report number [TID-8200(22nd Rev.)].

\section{NOTICE}

This report was prepared under the sponsorshıp of the U.S. Atomic Energy Commission. Neither the United States nor the U.S. Atomic Energy Commission, or any of their employees, contractors, subcontractors, or their employees, makes any warranty, express or implıed, or assumes any legal lıabılity or responsıbility for the accuracy, completeness, or usefulness of any information, apparatus, product, or process disclosed, or represents that its use would not infringe privately owned rights.

Printed in the United States of America

USAEC Division of Technical Information Extension, Oak Ridge, Tennessee 


\author{
NUCLEAR \\ REACTORS \\ BUILT, \\ BEING BUILT, \\ or \\ PLANNED
}

LEGAL NOTICE

This report was prepared as an account of work This the United States Atomic Energy the United states nor Commission, nor any of their emplo their employees, their contractors, subcontractors, or the or assumes any makes any warranty, express or implied, or assumes any legal liability or responsibility for the accuracy, cotus, pleteness or usefulness of any information, that its use product or process disclosed, or represents would not infringe privately owned rights.

\section{CIVILIAN REACTORS}

7 Central-Station Electric Power

12 Dual-Purpose Plants

12 Maritime Propulsion

12 Experimental Electric Power

13 Auxiliary Power (SNAP)

14 Space Propulsion (Rover)

15 General Irradiation Test

16 High-Power Research and Test

16 Safety Research and Test

17 General Research

19 University Research and Teaching

\section{PRODUCTION REACTORS}

2

Materials Production

21 Process Development

\section{MILITARY REACTORS}

22 Remote-Station Power

22 Propulsion (Naval)

24 Developmental Power

25 Test Reactors

25 Research Reactors

\section{REACTORS FOR EXPORT}

26 Central-Station Electric Power

27 Propulsion

27 General Irradiation Test

27 General Research

28 University Research and Teaching

\section{CRITICAL-ASSEMBLY FACILITIES}

29 Identification of Facilities

30 Experiments and Studies

36 Map Showing Locations of U.S. Nuclear Power Plants

37 Reactor Index 


\section{BLANK}




\section{FOREWORD}

This complation contains unclassified information about facilities bult, being built, or planned in the United States for domestic use or export as of June 30,1970, which are capable of sustaining a nuclear chain reaction. Information is presented in five parts, each of which is categorized by primary function or purpose. The major parts, namely, civllian, military, production, and export, as well as such categories as power and propulsion, are self-explanatory. Various classes of reactors withın these categones are defined as follows

CENTRAL-STATION NUCLEAR POWER PLANT A facility designed and constructed for operation on a utility system. The primary purpose of some of these plants is to demonstrate the economic and technical potential of future plants of the same general type, others, particularly those of the light-water type, are expected to be economically competitive with conventionally fueled plants in the geographic area in which they are located (Part I, Sec. 1A)

DUAL-PURPOSE PLANT A nuclear power facility designed, constructed, and operated for more than one primary purpose, for example, the production of nuclear materials and the generation of electricity or the use of reactor thermal energy for electrical generation and process-heat applications including desalting. (Part I, Sec 1B)

COMMERCIAL NUCLEAR POWER PLANT A nuclear facility constructed and operated under a license issued under Section 103 of the Atomic Energy Act of 1954 capable of producing steam for the generation of electricity, propulsion, and space- or process-heat applications upon which the AEC has made a finding of practical value withın the meaning of Section 102 of the Atomic Energy Act. (No reactors presently in the category)

EXPERIMENTAL POWER REACTOR A facility designed, engineered, constructed, and operated to test the technical feasibility of a concept or to provide the technical basis for a similar type nuclear power plant in a larger size Design flexibility permits changes to prove out various aspects of reactor technology including fuel and other components Power-conversion equipment may or may not be included as part of the facility. (Part I, Sec. 2A)

GENERAL IRRADIATION TEST REACTOR A reactor having (1) a thermal power level exceedıng 10,000 kW, (2) test loops or experimental facilities within, or in proximity to, the core, and (3) the use of nuclear radiation for testing the life or performance of reactor components as its major function. (Part I, Sec. 3A, and Part IV, Sec. 2A)

HIGH-POWER RESEARCH AND TEST REACTOR A reactor having a relatively high thermal power level ( $5000 \mathrm{~kW}$ or more) but not classed as a general irradiation test reactor (Part I, Sec. 3B)

SAFETY RESEARCH AND TEST REACTOR A reactor associated with a nuclear safety research or engineering-scale test program conducted for the purpose of developing basic design information or demonstrating safety characteristics of terrestrial and aerospace nuclear reactor systems. (Part I, Sec 3C)

RESEARCH REACTOR A reactor-excluding that located at a university - whose nuclear radiations are used primarily as a research tool for basic or applied research, and whose thermal power level is less than $5000 \mathrm{~kW}$. It may include facilities for testing reactor materials. (Part I, Sec 3D, Part III, Sec 3B, and Part IV, Sec. 2B)

UNIVERSITY RESEARCH AND TEACHING REACTOR A reactor located at a university and usually operated for the primary purpose of training in the operation and utilization of reactors and for instruction in reactor theory and performance. (Part I, Sec 3E, and Part IV, Sec. 2C)

SPECIAL TEST REACTOR A reactor designed for special testing purposes (Part III, Sec 3A)

CRITICAL FACILITY A reactor capable of sustaining a nuclear chain reaction operating at extremely low power (a few watts) and designed to determine a critical mass, neutron-flux distribution, and other characteristics of a flexible arrangement of nuclear fuel, construction materials, coolant, and other reactor components Fluid critical facilities are used to explore the critical masses of various concentrations of solutions in differing geometries. Metal critical assemblies are used to investigate the variations in heterogeneous cores. The tabulation of these facilities in Part V (pp 29-32) excludes those that have been operated and subsequently dismantled. 
The abbreviated listings in the principal nuclear contractor column refer to the technical organization assigned primary responsibility for design and/or fabrication of the reactor system The spelled out forms for those abbreviations as well as those for designers, shipbuilders, and facility operators, are given in the table on page

Startup dates refer to the year of first criticality Estımated startup dates based on the best avalable information are included for projects not yet in service The dates for non Commission projects are estımates announced by the sponsoring organizations

Reactors are listed as being operable under the following circumstances

1 Federal Government reactors - - when criticality is achieved

2 Non Federal Government reactors in the United States-when criticality is achieved or in the case of relatively low power svstems an operating license is issued by the Commission

3 Reactors for foreign locations - when criticality is acheved

Reactors are listed as being bult under the following circumstances

1 Tederal Government reactors-when ground is broken components are ordered or construction contract is awarded

2 Non Federal Government reactors in the United States- when the construction permit is issued by the Commission

3 Reactors for foreign locations-when an application for an export license is received by the Commission or when reliable information is received relating to the fabrication of reactor components

Reactors are listed as being planned under the following circumstances

1 Federal Government reactors - when publicly announced as a project planned for construction by the agency involved or the project is otherwise appropriately authorized

2 Non Federal Government reactors in the United States - when a public announcement that includes principal contractor and reactor type is made by the sponsoring organization or an application for a construction permit is received by the Commission

3 Reactors for foreign locations-when public announcement that includes principal contractor and reactor type is made or when the Commission receives information that a U S reactor manufacturer is proceeding with preconstruction design and development on the basis of a letter of intent

Reactors are listed as shut down or dismantled when the owner announces or verifies a decision to permanently shut down a faclity and does not intend to restart the reactor A reactor shut down owing to technical problems, extensive modifications, or refueling continues to be listed as operable

The Statistical Summary on page 5 excludes critical facilities All other categories are summarized Shutdown and dismantled reactors in these categories are included since such facilities have made significant contributions to reactor technology 


\section{STATISTICAL SUMMARY}

Shut down

Being or

Operable built Planned dismantled

\section{CIVILIAN REACTORS}

1. Power Reactors
A. Central-Station Electric Power
B. Dual-Purpose Plants
C. Propulsion (Maritime)

2. Experimental Power-Reactor Systems
A. Electric-Power Systems
B. Auxlliary Power (SNAP)
C. Space Propulsion (Rover)

3. Test, Research, and University Reactors

$\begin{array}{rrrr}15 & 54 & 32 & 6 \\ 1 & & 2 & \\ 1 & & & 21 \\ 3 & & & 8 \\ 1 & & & 18\end{array}$
A. General Irradiation Test
B. High-Power Research and Test
C. Safety Research and Test
D. General Research
E. Unıversity Research and Teachıng

\section{PRODUCTION REACTORS}

1. Materials Production

11

3

2. Process Development

\section{MlLITARY REACTORS}

1 Defense Power-Reactor Applications

A. Remote Installations

B. Propulsion (Naval)

$$
4
$$

104

25

2

2. Developmental Power

A. Electnc-Power Experıments and Prototypes

B. Propulsion Experiments and Prototypes

3. Test and Research
A. Test
B. Research

\section{REACTORS FOR EXPORT}

1. Power Reactors
A. Central-Station Electric Power
B. Propulsion
2. Test, Research, and Teaching
A. General Irradiation Test
B General Research
C Unıversity Research and Teachıng
2
1 


\section{LIST OF CONTRACTORS, DESIGNERS, SHIPBUILDERS, AND FACILITY OPERATORS FOR WHICH ABBREVIATIONS APPEAR IN TABLES}

AC
ACT
AG
AGN
AI
Alco
AMI
ANL
ANPD
ARSS
BAC
Bethlehem
Bettis
Blaw-Knox
BNL
BNW
B\&R
B\&W
CL
Comb
Convalr
Cook
CW
Daystrom
DOD
DOW
DUN
du Pont
Fosco

Allis-Chalmers $\mathrm{Mtg}$ Co

$\mathrm{ACl}$ Industries, Inc (reactor activities absorbed by $\mathrm{AC}$ )

Aerojet-General Corporation

Aerojet-General Nucleonics, formerly a subsidiary and now a

Division of Aerojet-General Corporation

Atomics International, d Division of North American Rockwell Corp Ako Products, Inc (reactor activities absorbed by $\mathrm{AC}$ )

AMF Atomics, Inc, a Division of American Machine \& Foundry Co Argonne National Laboratory, operated by the University of Chicago

Aircraft Nuclear Propulsion Department, General Electric Company

(name changed to Flight Propulsion Laboratory Department)

American Radıator \& Standard Sanitary Corp

Bendix Aviation Corp

Shipbuilding Division, Bethlehem Steel Co (now Quincy Division,

General Dynamics Corp )

Bettis Atomic Power Laboratory, operated by Westinghouse

Electric Corporation

Blaw-Knox Co

Brookhaven National Laboratory, operated by Associated

Universities, Inc

Battelle Northwest, a Division of Battelle Memorial Institute

Burns \& Roe, Inc

Babcock \& Wilcox Co

Clinton Laboratory of the Manhattan Engineer District

Combustion Engineering, Inc

Convalr Division, General Dynamics Corp

Nucledyne Co, a Division of Cook Electric Company

Curtiss-Wright Corporation

Daystrom, Inc

Department of Detense

The Dow Chemical Co, Rocky Flats Division

Douglas-United Nuclear, Inc

E I du Pont de Nemours \& Company, Inc

Ebasco Services, Inc
GNLC

GSA

HA

HKI

Hughes

IC

INC

Ingalls

Kaman

KAPL

$\mathrm{KI}$

LASL

Lockheed

Martın

Maxon

Met Lab

MINS

NASA

Newport News

NRDS

NRTS

NSA

NYSC

ORNL

Portsmouth

PPC

PRDC
General Nuclear Engineenng Corp (became a division of

Combustion Engineening, Inc, in 1964)

General Services Admınıstratıon

Hittman Associates

$\mathrm{H}$ K Ferguson Co

Hughes Aircraft $\mathrm{Co}$

Internuclear $\mathrm{Co}$

Idaho Nucledr Corporation

Ingalls Ship building Corp

Kaman Nuclear, a Division of Kaman Aircraft Corp

Knolls A tomic Power Laboratory, operated by General Electric Company

Kaiser Engineers, a Division of Henry $\mathbf{J}$ Ka1ser Co

Los Alamos Scientific Laboratory, operated by the University of Calıfornia

Lockheed Aircraft Corp

Martin Marietta Corp

Maxon Construction

Metallurgical Laboratory of the Manhattan Engineer District

Mare Island Naval Shipyard

National Aeronautics and Space Administration

National Bureau of Standards

Newport News Shipbuildıng \& Dry Dock Co

Nuclear Rocket Development Station

Naval Research Laboratory

National Reactor Testing Station

Nuclear Systems Associates

New York Shipbuilding Corp

Oak Ridge National Laboratory, operated by Unıon Carbide Corporation

Portsmouth Naval Shipyard

Phillips Petroleum Co

Power Reactor Development Company 
Electric Boat Electric Boat Division, General Dynamics Corp

FAST, Inc.

First A tomic Ship Transport, Inc , a subsidiary of American Lxport

Isbrandtsen Lines

The Fluor Corporation, Ltd

Foster Wheeler Corp

FW

GD (Quincy)

GGA

GE

GENMPO

GM
Quincy Division, General Dynamics Corp

Gult General A tomic Incorporated, a subsidiary of Gulf $\mathrm{O}_{1}$ Corporation (formerly General Atomic Division, General

Dynamics Corp )

General Electric Company

General Electric Nuclear Materials and Propulsion Operation General Motors Corp
P\&W

Sandid

San Francisco

Bay

TVA

UCLRL

UNC

WADCO

West
Pratt \& Whitney Aircraft Division, United Aircraft Corp.

Sandia Labordtories, operated by Sandia Corp, a subsidiary of

Western Electric Co.

San F rancisco Bay Naval Shipyard

Tennessee Valley Authority

University of Calıfornıa Lawrence Radıation Laboratory

United Nuclear Corporation, Development Division

Westinghouse Atomic Development $\mathrm{Co}$, a subsidiary of

Westinghouse Electric Corporation

Westinghouse Electric Corporation

\section{POWER REACTORS}

\section{A. Central-Station Electric Power}

\section{PART I CIVILIAN REACTORS (DOMESTIC)}

\begin{tabular}{|c|c|c|c|c|c|c|}
\hline \multirow[b]{2}{*}{ Name and/or owner } & \multirow[b]{2}{*}{ Location } & \multirow{2}{*}{$\begin{array}{l}\text { Principal } \\
\text { nuclear } \\
\text { contractor }\end{array}$} & \multirow[b]{2}{*}{ Type } & \multicolumn{2}{|c|}{ Power ${ }^{1}$} & \multirow[b]{2}{*}{$\begin{array}{c}\text { Start- } \\
\text { up }\end{array}$} \\
\hline & & & & $\begin{array}{c}\text { Plant, } \\
\text { net kW(e) }\end{array}$ & $\begin{array}{l}\text { Reactor, } \\
\mathrm{kW}(\mathbf{t})\end{array}$ & \\
\hline \multicolumn{7}{|l|}{ OPERABLE } \\
\hline Shippingport A tomic Power Station (AEC and Duquesne Light Co.) ${ }^{2}$ & Shıppıngport, $\mathrm{Pa}$ & West & Pressurized water & 90,000 & 505,000 & 1957 \\
\hline Dresden Nuclear Power Station, Unit 1 (Commonwealth Edison Co. $)^{3}$ & Morris, Ill. & $\mathrm{GL}$ & Bolling water & 200,000 & 700,000 & 1959 \\
\hline Yankee Nuclear Power Station (Yankee A tomic Electric Co ) ) $^{3,4}$ & Rowe, Mass & West. & Pressurized water & 175,000 & 600,000 & 1960 \\
\hline Big Rock Point Nuclear Plant (Consumers Power Co.) ${ }^{3,4}$ & Big Rock Point, Mich & GE & Bolling water & 70,300 & 240,000 & 1962 \\
\hline $\begin{array}{l}\text { Indian Point Station, Unit } 1 \text { (Consolidated Edison Co of New York, } \\
\text { Inc.) }\end{array}$ & Indian Point, N. Y. & $\mathrm{B} \& \mathrm{~W}$ & Pressurized water & 265,000 & 615,000 & 1962 \\
\hline $\begin{array}{l}\text { Ennco } \Gamma \text { ermı Atomic Power Plant (Power Reactor Development } \\
\text { Co.) }{ }^{3,4}\end{array}$ & Lagoona Beach, Mich. & PRDC & Sodium cooled, fast & 60,900 & 200,000 & 1963 \\
\hline Humboldt Bay Power Plant, Unıt 3 (Pactic Gas \& Electric Co $)^{3}$ & Eureka, Calıf & GE & Bolling water & 68,500 & 240,000 & 1963 \\
\hline $\begin{array}{l}\text { Peach Bottom Atomic Power Station, Unit } 1 \text { (Philadelphia Electric } \\
\text { Co.) }\end{array}$ & Peach Bottom, Pa. & GGA & $\begin{array}{l}\text { Gas cooled, graphite } \\
\text { moderated }\end{array}$ & 40,000 & 115,000 & 1966 \\
\hline $\begin{array}{l}\text { San Onofre Nuclear Generatıng Station, Unıt } 1 \text { (Southern Calıfornı } \\
\text { Edison and San Diego Gas \& Electric Co.) }\end{array}$ & San Clemente, Calıf. & West & Pressunzed water & 430,000 & $1,347,000$ & 1967 \\
\hline
\end{tabular}




\section{A. Central-Station Electric Power (Continued)}

\begin{tabular}{|c|c|c|c|c|c|c|c|}
\hline \multirow[b]{2}{*}{ Name and/or owner } & \multirow[b]{2}{*}{ Location } & \multirow{2}{*}{$\begin{array}{l}\text { Principal } \\
\text { nuclear } \\
\text { contractor }\end{array}$} & \multirow[b]{2}{*}{ Type } & \multicolumn{2}{|c|}{ Power $^{1}$} & \multirow[b]{2}{*}{$\begin{array}{l}\text { Start- } \\
\text { up }\end{array}$} & \multirow[b]{2}{*}{$\begin{array}{l}\text { Shut- } \\
\text { down }\end{array}$} \\
\hline & & & & $\begin{array}{l}\text { Plant, } \\
\text { net } k W(e)\end{array}$ & $\begin{array}{l}\text { Reactor, } \\
k W(t)\end{array}$ & & \\
\hline \multicolumn{8}{|l|}{ OPERABLE (Contınued) } \\
\hline $\begin{array}{l}\text { La Crosse Bolling Water Reactor (AEC and Darryland Power } \\
\text { Cooperative) }\end{array}$ & Genoa, Wis & $\mathrm{AC}$ & Bolling water & 50,000 & 165,000 & 1967 & \\
\hline $\begin{array}{l}\text { Haddam Neck Plant (Connecticut Yankee Atomic } \\
\text { Power (o) })^{3}\end{array}$ & Haddam Neck, Conn & West & Pressunzed water & 575,000 & $1,825,000$ & 1967 & \\
\hline $\begin{array}{l}\text { Oyster Creek Nuclear Power Plant, Unit } 1 \text { (Jersey Central } \\
\text { Power \& Light Co })^{3}\end{array}$ & Toms River, N J & GL & Bolling water & 530,000 & $1,600,000$ & 1969 & \\
\hline Nıne Mile Point Nuclear Station (N1agara Mohawk Power Corp) ${ }^{3}$ & Scriba, N Y & GE & Bollıng water & 500,000 & $1,538,000$ & 1969 & \\
\hline $\begin{array}{l}\text { Robert Emmett Gınna Nuclear Power Plant, Unıt } 1 \text { (Rochester } \\
\text { Gas \& Electric Co })^{3}\end{array}$ & Ontario, N Y & West & Pressunzed water & 420,000 & $1,300,000$ & 1969 & \\
\hline Dresden Nuclear Power Station, Unit 2 (Commonwealth Edison Co $)^{3}$ & Morris, Ill & GE & Bollıng water & 809,000 & $2,527,000$ & 1970 & \\
\hline \multicolumn{8}{|l|}{ BEING BUILT } \\
\hline $\begin{array}{l}\text { Millstone Nuclear Power Station, Unit } 1 \text { (Connecticut Light \& Power } \\
\text { Co, Hartford Electric Light Co, and Western Massachusetts } \\
\text { Electric Co })^{3}\end{array}$ & Waterford, Conn & GE & Bolling water & 652,100 & $2,011,000$ & 1970 & \\
\hline $\begin{array}{l}\text { Palisades Nuclear Power Station, Unit } 1 \text { (Consumers Power Co of } \\
\text { Michigan) }\end{array}$ & South Haven, Mich & Comb & Pressurized water & 700,000 & $2,212,000$ & 1970 & \\
\hline Dresden Nuclear Power Station, Unit 3 (Commonwealth Edıson Co $)^{3}$ & Morris, Ill & GE & Bollıng water & 809,000 & $2,527,000$ & 1970 & \\
\hline H B Robinson S E Plant, Unit 2 (Carolına Power \& Light Co $)^{3}$ & Hartsville, S C & West & Pressunzed water & 700,000 & $2,200,000$ & 1970 & \\
\hline Monticello Nuclear Generating Plant (Northern States Power Co ) ${ }^{3}$ & Monticello, Minn & GE & Bolling water & 545,000 & $1,670,000$ & 1970 & \\
\hline $\begin{array}{l}\text { Point Beach Nuclear Plant, Unit } 1 \text { (Wisconsin Electric Power } \\
\text { Co and Wisconsin Michigan Power Co })^{3}\end{array}$ & Two Creeks, Wis & West & Pressunzed water & 497,000 & $1,518,000$ & 1970 & \\
\hline Oconee Nuclear Station, Unit 1 (Duke Power Co $)^{3}$ & Seneca, S C & $\mathrm{B} \& \mathrm{~W}$ & Pressurized water & 841,120 & $2,452,000$ & 1970 & \\
\hline $\begin{array}{l}\text { Indian Point Station, Unit } 2 \text { (Consolidated Edison Co of New } \\
\text { York, Inc) }\end{array}$ & Indian Point, N Y & West & Pressurized water & 872,890 & $2,758,000$ & 1971 & \\
\hline Browns Ferry Nuclear Power Plant, Unit 1 (TVA) ${ }^{3}$ & Decatur, Ala & GE & Bollıng water & $1,064,500$ & $3,293,000$ & 1971 & \\
\hline $\begin{array}{l}\text { Peach Bottom Atomic Power Station, Unit } 2 \text { (Philadelphia } \\
\text { Electnc Co, Public Service Electric \& Gas Co, } \\
\text { Atlantıc City Electric Co, Delmarva Power \& Light Co })^{3}\end{array}$ & Peach Bottom, Pa & GE & Bolling water & $1,065,000$ & $3,294,000$ & 1971 & \\
\hline $\begin{array}{l}\text { Quad Cities Station, Unit } 1 \text { (Commonwealth Edison Co and } \\
\text { Iowa-Illınoss Gas and Electric Co })^{3}\end{array}$ & Cordova, Ill & GE & Boilıng water & 809,000 & $2,511,000$ & 1971 & \\
\hline Surry Power Station, Unit 1 (Virginia Electnc \& Power Co $)^{3}$ & Gravel Neck, Va & West & Pressunzed water & 780,000 & $2,441,000$ & 1971 & \\
\hline Zion Station, Unit 1 (Commonwealth Edison Co $)^{3}$ & Zion, Ill & West & Pressurized water & $1,050,000$ & $3,250,000$ & 1971 & \\
\hline Turkey Point Station, Unit 3 (Flonda Power \& Light Co $)^{3}$ & Turkey Point, Fla & West & Pressunzed water & 651,500 & $2,097,000$ & 1971 & \\
\hline $\begin{array}{l}\text { Vermont Yankee Generatıng Station (Vermont Yankee Nuclear } \\
\text { Power Corp ) }\end{array}$ & Vernon, $\mathrm{Vt}$ & GE & Bolling water & 513,900 & $1,593,000$ & 1971 & \\
\hline $\begin{array}{l}\text { Quad Cities Station, Unit } 2 \text { (Commonwealth Edison Co and } \\
\text { Iowa-Illinois Gas \& Electric Co })^{3}\end{array}$ & Cordova, Ill & GE & Bollung water & 809,000 & $2,511,000$ & 1971 & \\
\hline P1 1 ornm Station (Boston Edison Co ) & Plymouth, Mass & GE & Bollıng water & 654,000 & $1,998,000$ & 1971 & \\
\hline $\begin{array}{l}\text { Beach Nuclear Plant, Unit } 2 \text { (Wisconsin Electric } \\
\text { rower Co and Wisconsin Michigan Power Co })^{3}\end{array}$ & Two Creeks, Wis & West & Pressurized water & 497,000 & $1,518,000$ & 1971 & \\
\hline
\end{tabular}


St. Vrain Nuclear Generatıng Station (Public Service Co. of Colorado) ${ }^{3,4}$

Cooper Nuclear Station (Nebrasak Public Power District) ${ }^{3}$ Oconee Nuclear Station, Unit 2 (Duke Power Co.) ${ }^{3}$

Three Mile Island Station, Unit 1 (Metropolitan Edison Co.) ${ }^{3}$ Fort Calhoun Station, Unit 1 (Omaha Public Power District) ${ }^{3}$ Surry Power Station, Unit 2 (Virgmia Electric \& Power Co.) ${ }^{3}$ Salem Nuclear Generating Station, Unit 1

(Publıc Service Electric \& Gas Co., Phıladelphıa

Electric Co., Atlantic City Electric Co., Delmarva

Power \& Light Co.) ${ }^{3}$

Turkey Point Station, Unit 4 (Flonda Power \& Light Co.)

Diablo Canyon Nuclear Power Plant, Unit 1 (Pacific Gas \&

Electric Co. $)^{3}$

Prairie Island Nuclear Generating Plant, Unit 1 (Northern

States Power Co. $)^{3}$

Maine Yankee Atomic Power Plant (Mane Yankee Atomıc

Power Corp.) ${ }^{3}$

Browns Ferry Nuclear Power Plant, Unit 2 (Tennessee Valley Authonty) ${ }^{3}$

Kewaunee Nuclear Power Plant (Wisconsin Power \& Light

Co., Wisconsin Public Service Co., Madison Gas \& Electric Co. $)^{3}$

Crystal River Plant, Unit 3 (Flonda Power Corp.)

Peach Bottom Atomic Power Station, Unıt 3 (Philadelphıa

Electric Co., Public Service Electric \& Gas Co., Atlantıc

City Electric Company, Delmarva Power \& Light Co. $)^{3}$

Browns Ferry Nuclear Power Plant, Unit 3 (TVA) ${ }^{3}$

Rancho Seco Nuclear Generatıng Station, Unit 1 (Sacramento Municipal Utility District) ${ }^{3}$

Calvert Cliffs Nuçear Power Plant, Unit 1 (Baltımore Gas \& Electric Co.)

Edwin I. Hatch Nuclear Plant, Unit 1 (Georgia Power Co.) ${ }^{3}$

Donald C. Cook Nuclear Plant, Unit 1 (Indiana and Michigan

Electric Co. $)^{3}$

Oconee Nuclear Station, Unit 3 (Duke Power Co.) ${ }^{3}$

Beaver Valley Power Station, Unit 1 (Duquesne Light,

Ohı Edison Co., and Pennsylvania Power Co. $)^{3}$

Arkansas Nuclear One, Unit 1 (Arkansas Power \& Light Co $)^{3}$

Donald C. Cook Nuclear Plant, Unit 2 (Indiana and Michigan

Electric Co. $)^{3}$

Calvert Clıffs Nuclear Power Plant, Unit 2 (Baltımore Gas

\& Electric Co. $)^{3}$

Zion Station, Unit 2 (Commonwealth Edison Co.) ${ }^{3}$

Indian Point Station, Unit 3 (Consolidated Edison Co. of

New York, Inc.)

Salem Nuclear Generatıng Station, Unit 2 (Public Service Electric

\& Gas Co., Philadelphra Electric Co., Atlantıc City

Electric Co., Delmarva Power \& Laght Co.)

Three Mile Island Nuclear Station, Unit 2 (Jersey Central Power \& Light Co.) ${ }^{3}$
Brownville, Nebr.

Seneca, S. C.

Middle town, Pa.

Fort Calhoun, Nebr.

Gravel Neck, Va.

Salem, N. J

$B \& W$

moderated

Bolling water

Pressurized water

778,000

886,000

$2,381,000$

Pressurized water

Comb. Pressurized water

Pressurized water

457,400

780,000

$2,568,000$

$2,452,000$

$1,420,000$

Pressurized water

$1,050,000$

$2,441,000$

$3,250,000$

1971

1971

971

972

1972

Turkey Point, Fla.

West. Pressurzed wate

Pressurized water

651,500

$2,097,000$

$3,250,000$

1972

Diablo Canyon, Calif.

West.

Pressunzed water

530,000

$1,650,000$

1972

Wiscasset, Maine

Comb

Pressurized water

790,000

$2,440,000$

Decatur, Ala.

Borling water

$1,064,500$

$3,293,000$

Carlton, Wis

West.

Pressurized water

527,000

$1,650,000$

1972

Red Level, Fla.

Peach Bottom, $\mathrm{Pa}$

Pressurized water

858,000

$2,452,000$

1972

Decatur, Ala.

Clay Station, Calif.

GE

Lusby, Md.

Baxley, Ga

Brıdgman, Mich.

Seneca, S. C.

Midland, $\mathbf{P a}$

London, Ark

Bridgman, Mich.

Lusby, Md

Zion, Ill.

Indian Point, N, Y.

$1,065,000$

$3,294,000$

1972

Salem, N. J.

Borlıng water

B\&W Pressurized water

$1,064,500$

800,000

$3,293,000$

$2,452,000$

1972

Comb. Pressurized water

800,000

$2,450,000$

1972

GE Borling water

West.

Pressurnzed water

786,000

$2,436,000$

$3,250,000$

1972

B\&W $\quad$ Pressurized water

$, 054,000$

$2,568,000$

Pressurized water

886,000

$2,660,000$

1972

Pressunzed water

$2,452,000$

$3,250,000$

1973

West. Pressurized water

$1,060,000$

$2,450,000$

1973

West.- Pressunzed water

Pressurized water
Pressurized water

$3,250,000$

$3,025,000$

1973

1973

Pressurized water

$3,250,000$

1973

Middletown, $\mathrm{Pa}$

$\mathrm{B} \& \mathrm{~W}$

Pressurized water

810,000

$2,452,000$

1973 


\section{A. Central-Station Electric Power (Continued)}

\begin{tabular}{|c|c|c|c|c|c|c|c|}
\hline \multirow[b]{2}{*}{ Name and/or owner } & \multirow[b]{2}{*}{ Location } & \multirow{2}{*}{$\begin{array}{l}\text { Principal } \\
\text { nuclear } \\
\text { contractor }\end{array}$} & \multirow[b]{2}{*}{ Type } & \multicolumn{2}{|c|}{ Power ${ }^{1}$} & \multirow[b]{2}{*}{$\begin{array}{l}\text { Start- } \\
\text { up }\end{array}$} & \multirow[b]{2}{*}{$\begin{array}{l}\text { Shut- } \\
\text { down }\end{array}$} \\
\hline & & & & $\begin{array}{l}\text { Plant } \\
\text { Net kW(e) }\end{array}$ & $\begin{array}{l}\text { Reactor } \\
k W(t)\end{array}$ & & \\
\hline \multicolumn{8}{|l|}{ BEING BUILT (Contınued) } \\
\hline $\begin{array}{l}\text { Brunswick Steam Electric Plant, Unit } 2 \text { (Carolına Power } \\
\quad \& \text { Light Co.) }\end{array}$ & Southport, N. C. & GE & Bolling water & 821,000 & $2,436,000$ & 1973 & \\
\hline $\begin{array}{l}\text { Sequoyah Nuclear Power Plant, Unit } 1 \text { (Tennessee Valley } \\
\text { Authority) }\end{array}$ & Da1sy, Tenn. & West. & Pressurized water & $1,124,000$ & $3,423,000$ & 1973 & \\
\hline $\begin{array}{l}\text { Duane Arnold Energy Center, Unit } 1 \text { (lowa Electnc Light \& Power } \\
\text { Co., Central Iowa Power Cooperative and Corn Belt Power } \\
\text { Cooperative) }\end{array}$ & Palo, Iowa & GE & Bollıng water & 545,000 & $1,593,000$ & 1973 & \\
\hline $\begin{array}{l}\text { James A. FitzPatrick Nuclear Power Plant (Power Authonty } \\
\text { of the State of New York) }\end{array}$ & Scriba, N. Y. & GE & Bolling water & 821,000 & $2,436,000$ & 1973 & \\
\hline $\begin{array}{l}\text { Sequoyah Nuclear Power Plant, Unit } 2 \text { (Tennessee Valley } \\
\text { Authonty) }\end{array}$ & Dassy, Tenn. & West. & Pressurized water & $1,124,000$ & $3,423,000$ & 1974 & \\
\hline $\begin{array}{l}\text { Praurie Island Nuclear Generatıng Plant, Unit } 2 \text { (Northern } \\
\text { States Power Co ) }\end{array}$ & Red Wing, Mınn. & West & Pressurızed water & 530,000 & $1,650,000$ & 1974 & \\
\hline $\begin{array}{l}\text { Brunswick Steam Electric Plant, Unit } 1 \text { (Carolina Power \& } \\
\text { Light Co. })^{3}\end{array}$ & Southport, N. C. & GE & Bolling water & 821,000 & $2,436,000$ & 1975 & \\
\hline \multicolumn{8}{|l|}{ PLANNED } \\
\hline Hutchinson Island, Unit 1 (Florıda Power \& Light Co ) ${ }^{3}$ & Fort Pierce, Fla. & Comb. & Pressurnzed water & 800,000 & $2,440,000$ & 1973 & \\
\hline $\begin{array}{l}\text { Millstone Nuclear Power Station, Unit } 2 \text { (Connecticut Light \& } \\
\text { Power Co., Hartford Electric Light Co. and Western } \\
\text { Massachusetts Electric Co.) }\end{array}$ & Waterford, Conn. & Comb & Pressurnzed water & 828,000 & $2,560,000$ & 1973 & \\
\hline $\begin{array}{l}\text { North Anna Power Station, Unit } 1 \text { (Virginia Electric \& } \\
\text { Power Co. })^{3}\end{array}$ & Mineral, Va. & West. & Pressunzed water & 845,000 & $2,652,000$ & 1973 & \\
\hline $\begin{array}{l}\text { Diablo Canyon Nuclear Power Plant, Unit } 2 \text { (Pacific Gas \& } \\
\text { Electric Company) }\end{array}$ & Diablo Canyon, Calıf. & West. & Pressurized water & $1,060,000$ & $3,250,000$ & 1973 & \\
\hline $\begin{array}{l}\text { Enrico Ferm } 1 \text { Atomic Power Plant, Unit } 2 \text { (Detroit Edison } \\
\text { Company) }\end{array}$ & Lagoona Beach, Mıch. & GE & Bollung water & $1,123,000$ & $3,293,000$ & 1973 & \\
\hline $\begin{array}{l}\text { Trojan Nuclear Plant, Unit } 1 \text { (Portland General Electric Co., } \\
\text { Eugene Water \& Electric Board and Pacific Power \& } \\
\text { Light Co.) }\end{array}$ & Rainier, Oreg. & West. & Pressunzed water & $1,106,000$ & $3,423,000$ & 1974 & \\
\hline $\begin{array}{l}\text { Davis-Besse Nuclear Power Station (Toledo Edison Company } \\
\text { and Cleveland Electric Illumınatıng Company) }\end{array}$ & Oak Harbor, Ohio & B\&W & Pressurized water & 872,000 & $2,650,000$ & 1974 & \\
\hline Joseph M. Farley Nuclear Plant (Alabama Power Company) ${ }^{3}$ & Dothan, Ala & West. & Pressurized water & 829,000 & $2,652,000$ & 1974 & \\
\hline $\begin{array}{l}\text { North Anna Power Station, Unit } 2 \text { (Virginia Electric \& } \\
\text { Power Co. })^{3}\end{array}$ & Mineral, Va. & West. & Pressurnzed water & 845,000 & $2,652,000$ & 1974 & \\
\hline $\begin{array}{l}\text { Newbold Island Nuclear Generating Station, Unit } 1 \\
\text { (Public Service Electric \& Gas Co.) }\end{array}$ & Newbold Island, N. J. & GE & Bollıng water & $1,088,000$ & $3,293,000$ & 1974 & \\
\hline Limenck Generatıng Statıon, Unıt 1 (Philadelphıa Electrıc Co. $)^{3}$ & Pottstown, Pa. & GE & Bolling water & $1,065,000$ & $3,294,000$ & 1974 & \\
\hline $\begin{array}{l}\text { Wh } 1 \text { liam H. Zimmer Nuclear Power Station, Unit } 1 \\
\text { incinnatı Gas \& Electric Co., Columbus \& Southern }\end{array}$ & Moscow, Ohio & GE & Boilung water & 810,000 & $2,436,000$ & 1974 & \\
\hline
\end{tabular}


Pennsylvania Power \& Light Co, Unit 1

Shoreham Nuclear Power Station (Long Island Lighting Co.)

Willaam H. Zımmer Nuclear Power Station, Unit 2

(Cincinnatı Gas \& Electnc Co., Columbus \& Southern

Oho Electric Co., and Dayton Power \& Light Co. ${ }^{3}$

Willam B. McGuıre Nuclear Statıon, Unit 1 (Duke Power Co.)

Forked River Nuclear Generatıng Station, Unit 1

(Jersey Central Power \& Light Co.)

San Onofre Nuclear Generatıng Station, Unit 2

(Southern Calıforma Edison Co., and San Diego

Gas \& Electric Co.) ${ }^{3}$

Malıbu Nuclear Plant, Unıt 1 (Los Angeles Department of

Water \& Power) ${ }^{3,4}$

Bailly Generatıng Station (Northern Indiana Publıc Service Co.)

Carolina Power \& Light Co.

Newbold Island Nuclear Generating Station, Unit 2

(Public Service Electric \& Gas Co.) ${ }^{3}$

Edwin I. Hatch Nuclear Plant, Unit 2 (Georgia Power Co.)

Aguirre Nuclear Power Plant (Puerto Rico Water Resources

Authonty)

Arkansas Nuclear One, Unit 2 (Arkansas Power \& Light Co.)

Limenck Generatıng Statıon, Unıt 2 (Philadelphıa

Electric Co. $)^{3}$

San Onofre Nuclear Generatıng Statıon, Unıt 3 (Southern

Calıfornia Edison Co., and San Diego Gas \& Electric

$$
\mathrm{Co})^{3}
$$

LaSalle County Nuclear Station, Unit 1 (Commonwealth Edison Co )

LaSalle County Nuclear Station, Unit 2 (Commonwealth

Edison Co )

Consolidated Edison Co

Pennsylvania Power \& Light Co., Unit 2

Bell Station (New York State Gas \& Electric Co )

William B McGutre Nuclear Station, Unit 2 (Duke Power Co )

\section{SHUT DOWN OR DISMANTLED}

Hallam Nuclear Power Facility, Sheldon Station (AEC and

Consumers Public Power District ${ }^{4,7}$

Carolınas-Vırginia Tube Reactor (Carolınas-Virginıa

Nuclear Power Associates, Inc.) ${ }^{34}$

Piqua Nuclear Power Facility (AEC and City of Piqua) ${ }^{3} 4$

Bolling Nuclear Superheater Power Station (AEC and Puerto

Rico Water Resources Authority) ${ }^{34}$

Pathfinder Atomyc Power Plant (Northern States Power Co ) 3 4,9

Elk River Reactor (AEC and Rural Cooperative Power

Association) $)^{3,4,10}$
Bolling water

Cowans Ford Dam, N. C. West

Pressurzed water

$1,150,000$

$3,423,000$

Forked River, N. J. Comb.

Pressurized water

$1,129,000$

$3,390,000$

1975

San Clemente, Calıf.

Comb.

Pressurized water

$1,140,000 \quad 3,410,000$

Corral Canyon, Calıf. West. Pressurized water

462,000

$1,473,000$

1976

Dunes Acres, Ind

Bolling water

Bolling water

Bolling water

660,000

821,000

Newbold Island, N. J.

$1,088,000$

$1,931,000$

1976

Baxley, Ga.

GE

Boiling water

786,000

583,000

$3,293,000$

Puerto Rico

Pressurnzed water

$2,436,000 \quad 1976$

London, Ark.

Comb.

Pottstown, $\mathrm{P}$

GE

Pressurized water

950,000

$1,065,000$

$3,294,000$

1976

San Clemente, Calıf

Comb.

Pressurized water

$1,140,000$

$3,410,000$

1976

1976

Seneca, III

GE

Boilıng water

$1,100,000$

1976

Seneca, III.

Bolling water

$1,100,000$

Verplanck, N Y

GE

Lansing, N. Y.

Boiling water

Boilung water

Bolling water

$1,115,000$

$1,052,000$

838,000

Pressurized water

$1,150,000$

Cowans Tord Dam $N$ C

Hallam, Nebr

AI

Sodium graphite

75,000

17,000

65,000

11,400

45,500

1963

Pıqua, Ohıo

AI

Punta Higuera, P R

Comb.

Organic cooled and

16,500

50,000

1964

1968

S1oux Falls, S Dak

AC

integral nuclear

superheat

Boiling water,

58,500

190,000

1964

1968

Elk River, Minn

$\mathrm{AC}$

Bolling water

22,000

58,200

1962 


\section{B. Dual-Purpose Plants}

\begin{tabular}{|c|c|c|c|c|c|c|}
\hline \multirow[b]{2}{*}{ Name and/or owner } & \multirow[b]{2}{*}{ Location } & \multirow{2}{*}{$\begin{array}{c}\text { Principal } \\
\text { nuclear } \\
\text { contractor }\end{array}$} & \multirow[b]{2}{*}{ Type } & \multicolumn{2}{|c|}{ Power $^{1}$} & \multirow[b]{2}{*}{$\begin{array}{c}\text { Start } \\
\text { up }\end{array}$} \\
\hline & & & & $\begin{array}{c}\text { Plant, } \\
\text { net } k W(e)\end{array}$ & $\begin{array}{l}\text { Reactor, } \\
k W(t)\end{array}$ & \\
\hline \multicolumn{7}{|l|}{ OPERABLE } \\
\hline N Reactor (AEC and Washıngton Publıc Power Supply System) ${ }^{11}$ & Richland, Wash & DUN & Graphite & 790,000 & & 1963 \\
\hline \multicolumn{7}{|l|}{ PLANNED } \\
\hline $\begin{array}{l}\text { Midland Nuclear Power Plant, Unit } 1 \text { (Consumers Power Co of } \\
\text { Michigan) }\end{array}$ & Midland, Mich & B\&W & Pressurized water & 492,000 & $2,468,000$ & 1973 \\
\hline $\begin{array}{l}\text { Midland Nuclear Power Plant, Unit } 2 \text { (Consumers Power Co of } \\
\text { Michigan) }{ }^{3} 12\end{array}$ & Midland, Mich & $\mathrm{B} \& \mathrm{~W}$ & Pressurızed water & 818,000 & $2,468,000$ & 1974 \\
\hline
\end{tabular}

\section{Propulsion (Maritime)}

\begin{tabular}{|c|c|c|c|c|c|c|}
\hline Name and/or owner & Nuclear designer & Shupbuilder & Type & $\begin{array}{l}\text { Maxımum shaft } \\
\text { horsepower }\end{array}$ & Power, ${ }^{1}$ kW(t) & $\begin{array}{l}\text { Start- } \\
\text { up }\end{array}$ \\
\hline \multicolumn{7}{|l|}{ OPERABLE } \\
\hline $\begin{array}{l}\text { Nuclear Ship SAVANNAH (Maritume Admınistration- } \\
\text { operated by FAST, Inc ) }\end{array}$ & $\mathrm{B} \& \mathrm{~W}$ & NYSC & Pressurized water & 22,000 & 80,000 & 1961 \\
\hline
\end{tabular}

\section{EXPERIMENTAL POWER-REACTOR SYSTEMS}

\section{A. Electric-Power Systems}

\begin{tabular}{|c|c|c|c|c|c|c|c|c|}
\hline \multirow[b]{2}{*}{ Name (all owned by AEC except as noted) } & \multirow[b]{2}{*}{ Designation } & \multirow[b]{2}{*}{ Location } & \multirow{2}{*}{$\begin{array}{l}\text { Principal } \\
\text { nuclear } \\
\text { contractor }\end{array}$} & \multirow[b]{2}{*}{ Type } & \multicolumn{2}{|c|}{ Power ${ }^{1}$} & \multirow[b]{2}{*}{$\begin{array}{l}\text { Start- } \\
\text { up }\end{array}$} & \multirow[b]{2}{*}{$\begin{array}{l}\text { Shut- } \\
\text { down }\end{array}$} \\
\hline & & & & & $\begin{array}{c}\text { Plant, } \\
\text { net kW(e) }\end{array}$ & $\begin{array}{l}\text { Reactor, } \\
\text { kW(t) }\end{array}$ & & \\
\hline \multicolumn{9}{|l|}{ OPERABLE } \\
\hline $\begin{array}{l}\text { Saxton Nuclear Experımental Reactor Project } \\
\text { (Saxton Nuclear Experımental Corp })^{3}\end{array}$ & & Saxton, $\mathrm{Pa}$ & West & Pressurized water & 3,000 & 28,000 & 1962 & \\
\hline Expenmental Breeder Reactor No 2 & EBR-2 & NRTS, Idaho & ANL & Sodium cooled, fast & 16,500 & 62,500 & 1963 & \\
\hline $\begin{array}{l}\text { Southwest Expenmental Fast Oxide Reactor } \\
\text { (Southwest Atomic Energy Associates) }^{3}\end{array}$ & SEFOR & Strickler, Ark & GE & Sodıum cooled, fast & No elec & 20,000 & 1969 & \\
\hline \multicolumn{9}{|l|}{ SHUT DOWN OR DISMANTLED } \\
\hline Boulung Reactor Experument No 1 & BORAX-1 & NRTS, Idaho & ANL & Bouling water & No elec & 1,400 & 1953 & 1954 \\
\hline Yomogeneous Reactor Experiment No 1 & HRE-1 & Oak Ridge, Tenn & ORNL & $\begin{array}{l}\text { Aqueous homogeneous } \\
\text { solution }\left(\mathrm{UO}_{2} \mathrm{SO}_{4}\right)\end{array}$ & 140 & 1,000 & 1952 & 1954 \\
\hline
\end{tabular}


Alamos Power Reactor Expenment No 1

Borling Reactor Experıments ${ }^{14}$

Los Alamos Power Reactor Experıment No 2

Homogeneous Reactor Experiment No. 2

Organic Moderated Reactor Expenment ${ }^{15}$

Los Alamos Molten Plutonium Reactor Experiment

Experımental Beryllum Oxıde Reactor ${ }^{16}$

Vallecitos Boilng Water Reactor (General Electric Company and Pacific Gas \& Electric Co. ${ }^{3}$

Expenmental Breeder Reactor No $1^{17}$

Heavy Water Components Test Reactor

\section{Bollung Reactor Experiment No. 5}

Sodum Reactor Expenment (AEC and Southern Calıfornia Edison Co.) ${ }^{18}$

Fxperimental Gas-Cooled Reactor ${ }^{19}$

Expenmental Organıc Cooled Reactor ${ }^{20}$

ESADA Vallecitos Expenmental Superheat Reactor (Empire States Atomic Development Associates

and General Electric Company) ${ }^{3}$

Experimental Bolling Water Reactor ${ }^{21}$

Plutonium Recycle Test Reactor

Molten Salt Reactor Expenment ${ }^{22}$

Ultra High Temperature Reactor Experiment
LAPRE-1 Los Alamos, N. Mex. LASL

BORAX-2, NRTS, Idaho

3,4

LAPRE-2 Los Alamos, N. Mex.

HRE-2 Oak Ridge, Tenn

OMRE NRTS, Idaho

LAMPRE-1 Los Alamos, N. Mex.

EBOR

NRTS, Idaho

VBWR Pleasanton, Calıf

EBR-1 NRTS, Idaho

HWCTR Savannah River

Laboratory,

Aıken, S. C.

BORAX-5 NRTS, Idaho

SRE-PEP Santa Susana, Calıf.

EGCR Odk Ridge, Tenn

EOCR NRTS, Idaho

EVESR Pleasanton, Calıf.

EBWR Argonne, Ill.

PRTR Richland, Wash.

MSRE

Oak Ridge, Tenn.

UHTREX Los Alamos, N. Mex.
ANL

Aqueous homogeneous

(phosphoric acid)

Bolling water

Aqueous homogeneous (phosphoric acid)

Aqueous homogeneous solution $\left(\mathrm{UO}_{2} \mathrm{SO}_{4}\right)$

Organic cooled and moderated

Fast molten plutonium fueled, sodium cooled

Gas cooled, $\mathrm{BeO}$

moderated

Bolling water

GE

ANL

du Pont

Sodium cooled, fast

Pressurized heavy

water

ANL Bolling water, integral

nuclear superheat

Sodium graphite

Gas cooled, graphite

moderated

$\mathrm{KE}-\mathrm{AC}$

Organic cooled and

moderated

GE

Light-water moderated, superheater

Bolling water

Pressure tube, heavy-

water moderated

and cooled

ORNL

Single region, graphite

moderated

Helium cooled

LASL
No elec.

No elec

1,000

1959

1959

300

5,200

1957

1961

No. elec.

16,000

1957

1963

No elec.

1,000

1961

No elec

10,000

5,000

50,000

1957

1963

$\begin{array}{llll}150 & 1,400 & 1951 & 1964\end{array}$

No elec.

1,400
61,000

1951
1962

1964
1964

$2,500 \quad 20,000 \quad 1962 \quad 1964$

7,500

30,000

1957

1966

21,000

84,300

No elec.

40,000

No elec.

17,000

1963

1967

4,000

No elec.

100,000

85,000

1956

1960

1967

1969

No elec.

8,000

1965

1969

No elec.

$3,000 \quad 1969$

\section{B. Auxiliary Power (SNAP)}

\begin{tabular}{|c|c|c|c|c|c|c|c|c|}
\hline \multirow[b]{2}{*}{ Name (all owned by AEC except as noted) } & \multirow[b]{2}{*}{ Designation } & \multirow[b]{2}{*}{ Location } & \multirow{2}{*}{$\begin{array}{l}\text { Principal } \\
\text { nuclear } \\
\text { contractor }\end{array}$} & \multirow[b]{2}{*}{ Type } & \multicolumn{2}{|c|}{ Power $^{1}$} & \multirow[b]{2}{*}{$\begin{array}{l}\text { Start- } \\
\text { up }\end{array}$} & \multirow[b]{2}{*}{$\begin{array}{l}\text { Shut- } \\
\text { down }\end{array}$} \\
\hline & & & & & $\begin{array}{c}\text { Plant, } \\
\text { net } k W(e)\end{array}$ & $\begin{array}{l}\text { Reactor, } \\
\text { kW(t) }\end{array}$ & & \\
\hline \multicolumn{9}{|l|}{ OPERABLE } \\
\hline SNAP-2/10 A, TSF Shreldıng Experiment & SNAP-TSF & Oak Ridge, Tenn. & AI-ORNL & $\mathrm{NaK}$ cooled & & 10 & 1967 & \\
\hline
\end{tabular}




\section{B. Auxiliary Power (SNAP) (Continued)}

\begin{tabular}{|c|c|c|c|c|c|c|c|c|}
\hline \multirow[b]{2}{*}{ Name (all owned by AEC except as noted) } & \multirow[b]{2}{*}{ Designation } & \multirow[b]{2}{*}{ Location } & \multirow{2}{*}{$\begin{array}{l}\text { Principal } \\
\text { nuclear } \\
\text { contractor }\end{array}$} & \multirow[b]{2}{*}{ Type } & \multicolumn{2}{|c|}{ Power ${ }^{1}$} & \multirow[b]{2}{*}{$\begin{array}{l}\text { Start } \\
\text { up }\end{array}$} & \multirow[b]{2}{*}{$\begin{array}{l}\text { Shut } \\
\text { down }\end{array}$} \\
\hline & & & & & $\begin{array}{l}\text { Plant } \\
\text { Net kW(e) }\end{array}$ & $\begin{array}{c}\text { Reactor } \\
k W(t)\end{array}$ & & \\
\hline \multicolumn{9}{|l|}{ SHUT DOWN OR DISMANTLED } \\
\hline SNAP-2 Experımental Reactor & SER & Santa Susana, Calıf & AI & $\mathrm{NaK}$ cooled & No elec & 50 & 1959 & 1961 \\
\hline SNAP-2 Developmental System & S2DS & Santa Susana, Calıf & $\mathrm{AI}$ & $\mathrm{NaK}$ cooled & No elec & 50 & 1961 & 1963 \\
\hline SNAP-10A Flight System Ground Test No 1 & S10FS-1 & Santa Susana, Calıf & AI & NaK cooled & 05 & 39 & 1964 & 1964 \\
\hline SNAP-8 Experimental Reactor & S8ER & Santa Susana, Calıf & AI & NaK cooled & No elec & 600 & 1962 & 1965 \\
\hline SNAP-10A Flight System 23 & S10F S-4 & In orbit & AI & NaK cooled & 05 & 39 & 1965 & 1965 \\
\hline SNAP-10A Flight System Ground Test No 3 & S10F S-3 & Santa Susana, Calıf & AI & NaK cooled & 05 & 39 & 1964 & 1966 \\
\hline SNAP-10A Flight System & S10FS-5 & Santa Susana, Calff & AI & NaK cooled & 05 & 39 & (Spare) & \\
\hline SNAP-8, Developmental Reactor & S8DR & Santa Susana, Calif & AI & $\mathrm{NaK}$ cooled & & 600 & 1968 & 1969 \\
\hline
\end{tabular}

\section{Space Propulsion (Rover) ${ }^{24}$}

\begin{tabular}{|c|c|c|c|c|c|c|}
\hline Name (all owned by AEC except as noted) & Designation & Location & $\begin{array}{c}\text { Principal } \\
\text { nuclear } \\
\text { contractor }\end{array}$ & Type & $\begin{array}{c}\text { Power, } \\
\text { kW(t) }\end{array}$ & Operation \\
\hline \multicolumn{7}{|l|}{ BEING BUILT } \\
\hline Test Bed Reactor & Pewee-2 & NRDS, Nev & LASL & $\begin{array}{l}\text { Open cycle } \\
\text { liquid } \\
\text { hydrogen }\end{array}$ & & 1971 \\
\hline Fuel Element Test Bed & NF-1 & NRDS, Nev & LASL & $\begin{array}{l}\text { Open cycle } \\
\text { liquid } \\
\text { hydrogen }\end{array}$ & 50,000 & 1971 \\
\hline \multicolumn{7}{|l|}{ PLANNED } \\
\hline Fuel Element Test Reactor & Pewee-3 & NRDS, Nev & LASL & $\begin{array}{l}\text { Open cycle, liquid } \\
\text { hydrogen }\end{array}$ & & 1971 \\
\hline Fuel Element Test Reactor & Pewee-4 & NRDS, Nev & LASL & $\begin{array}{l}\text { Open cycle, liquid } \\
\text { hydrogen }\end{array}$ & & 1972 \\
\hline \multicolumn{7}{|l|}{ SHUT DOWN OR DISMANTLED } \\
\hline Nuclear Rocket Reactor Experiment & Kiw1-A & NRDS, Nev & LASL & $\begin{array}{l}\text { Open cycle, liquid } \\
\text { hydrogen }\end{array}$ & 70,000 & 1959 \\
\hline Nuclear Rocket Reactor Experıment & $\begin{array}{l}\text { Kiwl-A } \\
\text { Prime }\end{array}$ & NRDS, Nev & LASL & $\begin{array}{l}\text { Open cycle, hquid } \\
\text { hydrogen }\end{array}$ & 85,000 & 1960 \\
\hline Nuclear Rocket Reactor Exper'ment & KiW1-A3 & NRDS, Nev & LASL & $\begin{array}{l}\text { Open cycle, hquid } \\
\text { hydrogen }\end{array}$ & 100,000 & 1960 \\
\hline Nuclear Rocket Reactor Experıment & K1w1-B1A & NRDS, Nev & LASL & $\begin{array}{l}\text { Open cycle, hydrogen } \\
\text { gas cooled }\end{array}$ & 300,000 & 1961 \\
\hline lear Rocket Reactor Experment & Kiw1-B1B & NRDS, Nev & LASL & $\begin{array}{l}\text { Open cycle, liquid } \\
\text { hydrogen }\end{array}$ & 900,000 & 1962 \\
\hline
\end{tabular}




$\begin{array}{ll}\text { Kiwl-B4A } & \text { NRDS, Nev } \\ \text { Kıwl-B4D } & \text { NRDS, Nev } \\ \text { Kıwı-B4E } & \text { NRDS, Nev } \\ \text { NRX-A2 } & \text { NRDS, Nev } \\ \text { NRX A3 } & \text { NRDS, Nev } \\ \text { Phoebus 1A } & \text { NRDS, Nev } \\ \text { NRX-A4/EST } & \text { NRDS, Nev } \\ \text { NRX-A5 } & \text { NRDS, Nev } \\ \text { Phoebus 1B } & \text { NRDS, Nev } \\ \text { NRX-A6 } & \text { NRDS, Nev } \\ \text { Phoebus 2A } & \text { NRDS, Nev } \\ \text { Pewee-1 } & \text { NRDS, Nev } \\ \text { XE-Prime } & \text { NRDS, Nev }\end{array}$

\begin{tabular}{|c|c|}
\hline LASL & $\begin{array}{l}\text { Open cycle, liquid } \\
\text { hydrogen }\end{array}$ \\
\hline LASL & $\begin{array}{l}\text { Open cycle, liquid } \\
\text { hydrogen }\end{array}$ \\
\hline LASL & $\begin{array}{l}\text { Open cycle, liquid } \\
\text { hydrogen }\end{array}$ \\
\hline AG-West & $\begin{array}{l}\text { Open cycle, hquid } \\
\text { hydrogen }\end{array}$ \\
\hline AG West & $\begin{array}{l}\text { Open cycle, hquid } \\
\text { hydrogen }\end{array}$ \\
\hline LASL & $\begin{array}{l}\text { Open cycle, liquid } \\
\text { hydrogen }\end{array}$ \\
\hline AG-West & $\begin{array}{l}\text { Open cycle, liquid } \\
\text { hydrogen }\end{array}$ \\
\hline AG-West & $\begin{array}{l}\text { Open cycle, liquid } \\
\text { hydrogen }\end{array}$ \\
\hline LASL & $\begin{array}{l}\text { Open cycle, liquid } \\
\text { hydrogen }\end{array}$ \\
\hline AG-West & $\begin{array}{l}\text { Open cycle, liquid } \\
\text { hydrogen }\end{array}$ \\
\hline LASL & $\begin{array}{l}\text { Open cycle, hquid } \\
\text { hydrogen }\end{array}$ \\
\hline LASL & $\begin{array}{l}\text { Open cycle, hquid } \\
\text { hydrogen }\end{array}$ \\
\hline AG-West & $\begin{array}{l}\text { Open cycle, hquid } \\
\text { hydrogen }\end{array}$ \\
\hline
\end{tabular}
(n)

Nuclear Rocket Reactor Experıment

Nuclear Rocket Engıne Reactor Experiment (NERVA)

Nuclear Rocket Engine Reactor Experiment (NERVA)

Nuclear Rocket Reactor Experiment

Nuclear Rocket Reactor Engne System Test (NERVA)

Nuclear Rocket Engine Reactor Expenment (NERVA)

Nuclear Rocket Reactor Experıment

Nuclear Rocket Engme Experiment

(NERVA)

Nuclear Rocket Reactor Experiment

Fuel Element Test Reactor

Ground Experımental Engine Experıment hydrogen

$950,000 \quad 1964$

$1,100,000 \quad 1964$

$1,120,000 \quad 1965$

$1,070,000 \quad 1965$

$1,190,000 \quad 1966$

$1,100,000 \quad 1966$

$1,400,000 \quad 1967$

$1,100,000 \quad 1967$

$4,200,000 \quad 1968$

$514,000 \quad 1968$

$1,100,000 \quad 1968$

\section{TEST, RESEARCH, AND UNIVERSITY REACTORS}

\section{A. General Irradiation Test}

\begin{tabular}{|c|c|c|c|c|c|c|c|c|}
\hline Name and/or owner & Designation & Location & $\begin{array}{c}\text { Principal } \\
\text { nuclear contractor }\end{array}$ & Operator & Type & $\begin{array}{c}\text { Power, }^{1} \\
\text { kW(t) }\end{array}$ & $\begin{array}{l}\text { Start- } \\
\text { up }\end{array}$ & $\begin{array}{l}\text { Shut- } \\
\text { down }\end{array}$ \\
\hline \multicolumn{9}{|l|}{ OPERABLE } \\
\hline Engineering Test Reactor (AEC) & ETR & NRTS, Idaho & $\mathbf{K E}-\mathrm{GE}$ & INC & Tank & 175,000 & 1957 & \\
\hline General Electric Test Reactor ${ }^{3}$ & GETR & Pleasanton, Calıf & Owner & Owner & Tank & 50,000 & 1958 & \\
\hline Plum Brook Reactor Facllty (NASA) ${ }^{3}$ & NASA-TR & Sandusky, Ohıo & NASA & NASA & Tank & 60,000 & 1961 & \\
\hline Advanced Test Reactor (AEC) & ATR & NRTS, Idaho & Ebasco-B\&W & INC & Tank & 250,000 & 1968 & \\
\hline \multicolumn{9}{|l|}{ PLANNED } \\
\hline Fast Flux Test Facility (AEC) & FFTF & Richland, Wash & WADCO & WADCO & $\begin{array}{l}\text { Sodium } \\
\text { cooled }\end{array}$ & 400,000 & 1974 & \\
\hline \multicolumn{9}{|l|}{ SHUT DOWN OR DISMANTLED } \\
\hline Westınghouse Testıng Reactor ${ }^{3}$ & WTR & Waltz Mill, $\mathrm{Pa}$ & Owner & Owner & Tank & 60,000 & 1959 & 1962 \\
\hline Matenals Testıng Reactor $(\mathrm{AEC})^{25}$ & MTR & NRTS, Idaho & ORNL-ANL-Blaw-Knox & INC & Tank & 40,000 & 1952 & 1970 \\
\hline
\end{tabular}




\section{B. High-Power Research and Test}

\begin{tabular}{|c|c|c|c|c|c|c|c|}
\hline Name and/or owner & Designation & Location & $\begin{array}{c}\text { Principal } \\
\text { nuclear contractor }\end{array}$ & Type & $\begin{array}{c}\text { Power, } \\
\text { kW(t) }\end{array}$ & $\begin{array}{c}\text { Start- } \\
\text { up }\end{array}$ & $\begin{array}{l}\text { Shut- } \\
\text { down }\end{array}$ \\
\hline \multicolumn{8}{|l|}{ OPERABLE } \\
\hline Argonne Research Reactor (AEC) & $\mathrm{CP}-5$ & Argonne, Ill. & ANL & Heavy water & 5,000 & 1954 & \\
\hline Omega West Reactor (AEC) & OWR & Los Alamos, N. Mex. & LASL & Tank & 8,000 & 1956 & \\
\hline Industrial Reactor Laboratories, Inc. ${ }^{3}$ & & Plainsboro, N. J. & AMF & Pool & 5,000 & 1958 & \\
\hline Oak Rıdge Research Reactor (AEC) & ORR & Oak Ridge, Tenn. & ORNL & Tank & 30,000 & 1958 & \\
\hline $\begin{array}{l}\text { Brookhaven Medical Research Reactor } \\
\text { (AEC) }\end{array}$ & MRR & Upton, N Y. & Daystrom & Tank & 5,000 & 1959 & \\
\hline Union Carbide Corp. ${ }^{3}$ & UCNR & Sterlıng Forest, N. Y. & $\mathrm{AMF}$ & Pool & 5,000 & 1961 & \\
\hline $\begin{array}{l}\text { Babcock \& Wilcox Nuclear Development } \\
\text { Center Test Reactor }{ }^{3}\end{array}$ & BAWTR & Lynchburg, Va. & Owner & Pool & 6,000 & 1964 & \\
\hline Ames Laboratory Research Reactor (AEC) & ALRR & Ames, Iowa & AMF & Heavy water & 5,000 & 1965 & \\
\hline $\begin{array}{l}\text { Brookhaven High Flux Beam Research } \\
\text { Reactor (AEC) }\end{array}$ & HFBR & Upton, N. Y. & BNL & Heavy water & 40,000 & 1965 & \\
\hline High Flux Isotope Reactor (AEC) & HFIR & Oak Ridge, Tenn. & ORNL & Tank flux trap & 100,000 & 1965 & \\
\hline National Bureau of Standards ${ }^{3}$ & NBSR & Gaithersburg, Md. & NBS-B\&R & Heavy water & 10,000 & 1967 & \\
\hline \multicolumn{8}{|l|}{ SHUT DOWN OR DISMANTLED } \\
\hline Brookhaven Research Reactor (AEC) & BGRR & Upton, N. Y. & $\mathrm{HKF}$ & Graphite & 20,000 & 1950 & 1969 \\
\hline Sandıa Engineerıng Reactor (AEC) & SER & Sand1a Base, N. Mex & Sandia & Tank & 5,000 & 1962 & 1970 \\
\hline
\end{tabular}

\section{Safety Research and Test}

\begin{tabular}{|c|c|c|c|c|c|c|c|}
\hline Name (all owned by AEC) & Designation & Location & $\begin{array}{c}\text { Principal } \\
\text { nuclear contractor }\end{array}$ & Type & $\begin{array}{c}\text { Power, }{ }^{1} \\
\text { kW(t) }\end{array}$ & $\begin{array}{l}\text { Start- } \\
\text { up }\end{array}$ & $\begin{array}{l}\text { Shut- } \\
\text { down }\end{array}$ \\
\hline \multicolumn{8}{|l|}{ OPERABLE } \\
\hline Special Power Excursion Reactor Test No. 4 & SPERT-4 & NRTS, Idaho & INC & Pool & Transient & 1962 & \\
\hline Transient Reactor Test & TREAT & NRTS, Idaho & ANL & Graphite & Transient & 1959 & \\
\hline Intrinsic Subcritıcal Assembly ${ }^{26}$ & SNAPTRAN-1 & Santa Susana, Calıf. & AI & $\begin{array}{l}\text { Be-reflected } \\
\text { SNAP-10A }\end{array}$ & Transient & 1968 & \\
\hline \multicolumn{8}{|l|}{ BEING BUILT } \\
\hline Power-Burst Facility & PBF & NRTS, Idaho & INC & Tank & $\begin{array}{l}\text { Transient, } \\
20,000\end{array}$ & 1971 & \\
\hline Loss of Fluid Test & LOFT & NRTS, Idaho & INC & $\begin{array}{l}\text { Pressurized } \\
\text { water }\end{array}$ & 50,000 & 1973 & \\
\hline $\begin{array}{l}\text { SHUT DOWN OR DISMANTLED } \\
\text { SNAP-10A Transient Test No. } 3^{27}\end{array}$ & SNAPTRAN-3 & NRTS, Idaho & $\mathrm{PPC}-\mathrm{AI}$ & $\begin{array}{l}\mathrm{H}_{2} \mathrm{O} \text {-reflected } \\
\text { SNAP-10A }\end{array}$ & Transient & 1964 & 1964 \\
\hline
\end{tabular}


Cecial Power Excursion Reactor Test No. 1 Kiw1 Transient Test Reactor

Special Power Excursion Reactor Test No. $2^{28}$

SNAP-10A Transient Test No. $2^{27}$

Kunetic Experiment on Water Boilers

Special Power Excursion Reactor Test No. 3
SPERT-1 NRTS, Idaho

KIW1-TNT

SPERT-2

NRDS, Nev.

NRTS, Idaho

SNAPTRAN-2

NRTS, Idaho

KEWB

SPERT-3
Santa Susana, Calıf NRTS, Idaho

\section{PPC}

LASL

PPC

AI-PPC

AI

PPC
Open tank

Kiw1/NERVA

Pressurized

water

Be-reflected

SNAP-10A

Homogeneous

Pressurized

water
Transient

Transient

Transient

Transient

Transient Transient

\section{General Research}

\begin{tabular}{|c|c|c|c|c|c|c|c|}
\hline Name and/or owner & Desıgnatıon & Location & $\begin{array}{l}\text { Principal } \\
\text { nuclear contractor }\end{array}$ & Type & $\begin{array}{c}\text { Power, }^{1} \\
k W(t)\end{array}$ & $\begin{array}{l}\text { Start- } \\
\text { up }\end{array}$ & $\begin{array}{l}\text { Shut- } \\
\text { down }\end{array}$ \\
\hline \multicolumn{8}{|l|}{ OPERABLE } \\
\hline Bulk Shielding Reactor (AEC) ${ }^{29}$ & BSR & Oak Ridge, Tenn & ORNL & Pool & 2,000 & 1950 & \\
\hline Los Alamos Water Boller (AEC) & SUPO & Los Alamos, N. Mex. & LASL & Homogeneous & 25 & 1950 & \\
\hline $\begin{array}{l}\text { North American Rockwell Water Boller } \\
\text { Neutron Source }(\mathrm{AEC})^{\mathbf{3 0}}\end{array}$ & AE-6(WBNS) & Santa Susana, Calıf. & $\mathrm{AI}$ & Homogeneous & 3 & 1952 & \\
\hline Physical Constants Test Reactor (AEC) & PCTR & Richland, Wash. & BNW & Graphite & 01 & 1955 & \\
\hline Thermal Test Reactor No. 2 (AEC) & TTR-2 & Richland, Wash & $\mathrm{BNW}$ & Graphite & 0.1 & 1955 & \\
\hline Los Alamos HYDRO Reactor (AEC) & HYDRO & Los Alamos, N. M. & LASL & & & 1956 & \\
\hline Battelle Memorial Institute ${ }^{3}$ & BRR & West Jefferson, Ohio & $\mathrm{AMF}$ & Pool & 2,000 & 1956 & \\
\hline Argonne National Laboratory (AEC) & AGN-201-108 & Argonne, Ill. & $\mathrm{AGN}$ & Homog solid & Neglig. & 1957 & \\
\hline $\begin{array}{l}\text { Argonne Nuclear Assembly for University } \\
\text { Training (AEC) }\end{array}$ & $\begin{array}{r}\text { Argonaut } \\
(\mathrm{CP}-11)\end{array}$ & Argonne, Ill. & ANL & Graphite/water & 10 & 1957 & \\
\hline General Electnc Nuclear Test Reactor ${ }^{3}$ & NTR & Pleasanton, Calıf. & GE & Light water & 100 & 1957 & \\
\hline Livermore Pool Type Reactor (AEC) & LPTR & Livermore, Calıf & FW & Tank & 3,000 & 1957 & \\
\hline Argonne Thermal Source Reactor (AEC) & ATSR & Argonne, Ill & ANL & Thermal & 10 & 1957 & \\
\hline North American Rockwell Corp ${ }^{3}$ & $\mathrm{~L}-77$ & Canoga Park, Calıf. & AI & Homogeneous & Neglig. & 1958 & \\
\hline Babcock \& Wilcox Lynchburg Pool Reactor ${ }^{3}$ & LPR & Lynchburg, Va. & Owner & Pool & 1,000 & 1958 & \\
\hline $\begin{array}{l}\text { Gulf General Atomic Incorporated, TRIGA-Mk I } \\
\text { Prototype Reactor } 3, \mathbf{3 1}\end{array}$ & TRIGA-Mk I & La Jolla, Calif. & GGA & $\mathrm{U}-\mathrm{Zr}$ hydride & 250 & 1958 & \\
\hline Pawlıng Research Reactor (United Nuclear Corp) ${ }^{3}$ & PRR & Pawling, N. Y. & UNC & Light water & Neglig. & 1958 & \\
\hline $\begin{array}{l}\text { Radiation Effects Reactor (Lockheed Aircraft } \\
\text { Corp.) }\end{array}$ & RER & Dawsonville, Ga. & Lockheed & Pool & 3,000 & 1958 & \\
\hline Fast Source Reactor (AEC) & AFSR & NRTS, Idaho & ANL & Fast & 1 & 1959 & \\
\hline Omaha Veterans Admınıstration Hospital ${ }^{3}$ & TRIGA-Mk I & Omaha, Nebr & GGA & $\mathrm{U}-\mathrm{Zr}$ hydrude & 18 & 1959 & \\
\hline $\begin{array}{l}\text { Gulf General A tomic Incorporated, Advanced } \\
\text { TRIGA-Mk F Prototype Reactor }{ }^{3,33}\end{array}$ & TRIGA-Mk F & La Jolla, Calif & Owner & $\mathrm{U}-\mathrm{Zr}$ hydnde & 1,500 & 1960 & \\
\hline Shield Test and Irradiation Reactor (AEC) ${ }^{34}$ & STIR & Santa Susana, Calıf & $\mathrm{AI}$ & Pool & 1,000 & 1961 & \\
\hline Health Physics Research Reactor (AEC) ${ }^{35}$ & HPRR & Oak Ridge, Tenn & ORNL & Fast burst & 10 & 1962 & \\
\hline NASA Mock-Up Keactor ${ }^{3}$ & MUR & Sandusky, Ohı & Lockheed & Light water, pool & 100 & 1963 & \\
\hline $\begin{array}{l}\text { Northrop Corporate Laboratories (Space } \\
\text { Radiation Laboratory) } \\
3,31\end{array}$ & TRIGA-Mk F & Hawthorne, Calif. & GGA & $\mathbf{U}-\mathrm{Zr}$ hydride & 1,000 & 1963 & \\
\hline
\end{tabular}


D. General Research (Continued)

\begin{tabular}{|c|c|c|c|c|c|c|c|}
\hline Name and/or owner & Designation & Location & $\begin{array}{l}\text { Principal } \\
\text { nuclear contractor }\end{array}$ & Type & $\begin{array}{c}\text { Power }^{1} \\
\text { kW(t) }\end{array}$ & $\begin{array}{l}\text { Start- } \\
\text { up }\end{array}$ & $\begin{array}{l}\text { Shut- } \\
\text { down }\end{array}$ \\
\hline \multicolumn{8}{|l|}{ OPERABLE (Contınued) } \\
\hline USAEC European-Asıan Exhıbit Program ${ }^{36}$ (AEC) & & & Lockheed & Pool & 10 & 1963 & \\
\hline Biological Research Reactor (AEC) & JANUS & Argonne, Ill & ANL & Tank & 200 & 1964 & \\
\hline Rhode Island Nuclear Science Center ${ }^{3}$ & & Fort Kearney, R. I. & GE & Pool & 2,000 & 1964 & \\
\hline AGN Industrial Reactor ${ }^{3}$ & AGNIR & San Ramon, Calıf. & AGN & Pool-TRIGA core & 250 & 1965 & \\
\hline $\begin{array}{l}\text { Gulf General Atomic Incorporated, TRIGA-Mk III } \\
\text { Prototype Reactor }{ }^{3}\end{array}$ & TRIGA-Mk III & La Jolla, Calıf. & Owner & $\mathrm{U}-\mathrm{Zr}$ hydride & 1,500 & 1965 & \\
\hline Sandia Pulsed Reactor II (AEC) & SPR-II & Sandia Base, N Mex. & Sandıa & Prompt burst & Transient & 1967 & \\
\hline Annular Core Pulsed Reactor (AEC) & TRIGA-ACPR & Sandia Base, N Mex & GGA & $\mathrm{U}-\mathrm{Zr}$ hydride & Transient & 1967 & \\
\hline Dow Chemical Co. ${ }^{3}$ & TRIGA-Mk I & Midland, Mich. & GGA & $\mathrm{U}-\mathrm{Zr}$ hydride & 100 & 1967 & \\
\hline High Temperature Lattice Test Reactor (AEC) & HTLTR & Richland, Wash. & PNL & Graphite & 2 & 1967 & \\
\hline Accelerator Pulsed Fast Critıcal Assembly ${ }^{3,37}$ & APF A-III & La Jolla, Calıf. & GGA & Fast & 1 & 1967 & \\
\hline $\begin{array}{l}\text { U. S. Geological Survey Laboratory }{ }^{3,31} \text { (Depart- } \\
\text { ment of the Interior) }\end{array}$ & TRIGA-Mk I & Denver, Colo. & GGA & $\mathrm{U}-\mathrm{Zr}$ hydride & 1,000 & 1969 & \\
\hline $\begin{array}{l}\text { USAEC Latın American Demonstration Reactor } \\
\text { Center }^{38} \text { (AEC) }\end{array}$ & & & Lockheed & Pool & 10 & 1969 & \\
\hline \multicolumn{8}{|l|}{ SHUT DOWN OR DISMANTLED } \\
\hline $\begin{array}{l}\text { Chicago P1le 1, rebuilt as CP-2 (Manhattan } \\
\text { Engineer District - AEC) }\end{array}$ & $\mathrm{CP}-2$ & Chicago, Ill. & Met Lab. & Graphite & $0.2-2$ & 1942 & 1954 \\
\hline Oak Ridge Graphite Reactor (AEC) & $X-10$ & Oak Ridge, Tenn. & $\mathrm{CL}$ & Graphite & 3,800 & 1943 & 1963 \\
\hline $\begin{array}{l}\text { Argonne CP-3, rebuilt as CP- } 3^{\prime} \\
\text { (Manhattan Engineer District-AEC) }\end{array}$ & $\mathrm{CP}^{-3^{\prime}}$ & Palos Park, Ill. & Met. Lab. & Heavy water & 300 & 1944 & 1954 \\
\hline Los Alamos Water Boiler (AEC) & HYPO & Los Alamos, N Mex. & LASL & Homogeneous & 5.5 & 1944 & 1950 \\
\hline Los Alamos Fast Reactor (AEC) & Clementıne & Los Alamos, N. Mex. & LASL & $\begin{array}{l}\text { Fast, plutonium fuel, } \\
\text { mercury cooled }\end{array}$ & 25 & 1946 & 1953 \\
\hline Livermore Water Borler (AEC) & LIWB & Livermore, Calıf. & AI & Homogeneous & 0.5 & 1953 & 1961 \\
\hline $\begin{array}{l}\text { Illunois Institute of Technology Research } \\
\text { Institute (Armour Research Foundation) }\end{array}$ & $\operatorname{ARR}(L-54)$ & Chicago, Ill. & AI & Homogeneous & 75 & 1956 & 1967 \\
\hline Atomics International ${ }^{3}$ & $\mathrm{~L}-47$ & Canoga Park, Calif. & AI & Homogeneous & Neglig. & 1957 & 1958 \\
\hline Amencan Radiator \& Standard Sanitary Corp. ${ }^{40}$ & UTR-1 & Mountain View, Calif. & ARSS & Graphite/water & Neglig. & 1958 & 1960 \\
\hline $\begin{array}{l}\text { The Curtiss-Wright Nuclear Research Laboratory } \\
\text { of the Commonwealth of Pennsylvania }\end{array}$ & & Quehanna, Pa. & Owner & Pool & 1,000 & 1958 & 1966 \\
\hline Lockheed Aircraft Corp. & & Dawsonville, Ga. & Lockheed & Pool & Neglig. & 1960 & 1960 \\
\hline $\begin{array}{l}\text { Gulf General Atomic Incorporated (World Agncultural } \\
\text { Faur-U S. Exhibit Reactor) }\end{array}$ & TRIGA-Mk II & San Diego, Calıf. & GGA & $\mathrm{U}-\mathrm{Zr}$ hydride & 50 & 1960 & 1960 \\
\hline $\begin{array}{l}\text { UTR Test Reactor (Amencan Radiator \& Standard } \\
\text { Sanitary Corp ) }\end{array}$ & & Mountain View, Calif & Owner & Graphite/water & Neglig. & 1961 & 1963 \\
\hline $\begin{array}{l}\text { Louisiana State University Nuclear Science Center } \\
\text { (Sandia Nuclear Assembly for Reactor } \\
\text { Experiments) }\end{array}$ & SNARE & Baton Rouge, La. & Sand1a & Pool & 1 & 1965 & 1966 \\
\hline $\begin{array}{l}\text { erojet-General Corporation } \\
\text { andia Pulsed Reactor (AEC) }\end{array}$ & $\begin{array}{l}\text { AGN-201P-103 } \\
\text { SPR }\end{array}$ & $\begin{array}{l}\text { San Ramon, Calıf } \\
\text { Sandia Base, N. Mex. }\end{array}$ & $\begin{array}{l}\text { AGN } \\
\text { Sandia }\end{array}$ & $\begin{array}{l}\text { Homog. solıd } \\
\text { Prompt burst }\end{array}$ & Neglig. & 1957 & $\begin{array}{l}1966 \\
1967\end{array}$ \\
\hline
\end{tabular}


Low Intensity Test Reactor (AEC)

LITR

\section{E. University Research and Teaching}

(Footnote 3 applies to all reactors in this section)

\begin{tabular}{|c|c|c|c|c|c|c|c|}
\hline Name and/or owner & Designation & Location & $\begin{array}{c}\text { Principal } \\
\text { nuclear contractor }\end{array}$ & Type & $\begin{array}{c}\text { Power, }^{1} \\
\text { kW(t) }\end{array}$ & $\begin{array}{l}\text { Start- } \\
\text { up }\end{array}$ & $\begin{array}{l}\text { Shut- } \\
\text { down }\end{array}$ \\
\hline \multicolumn{8}{|l|}{ OPERABLE } \\
\hline Texas A\&M University & AGN-201-106 & College Station, Tex. & AGN & Homog. solid & Neglig. & 1957 & \\
\hline Catholıc Unıversity of America & AGN-201-101 & Washington, D. C. & AGN & Homog. solid & Neglig. & 1957 & \\
\hline Colorado State University & $A G N-201-109$ & Fort Collıns, Colo. & $\mathrm{AGN}$ & Homog. solid & Neglig & 1957 & \\
\hline $\begin{array}{l}\text { Oklahoma State University of Agniculture and } \\
\text { Applied Science }\end{array}$ & AGN-201-102 & Stillwater, Okla. & AGN & Homog. solid & Neglig. & 1957 & \\
\hline Georg1a Institute of Technology 46 & AGN-201-104 & Atlanta, Ga. & AGN & Homog. solid & Neglig & 1957 & \\
\hline University of New Mexico 47 & AGN-201M-112 & Albuquerque, N. Mex & AGN & Homog. solid & Neglig. & 1957 & \\
\hline Unıversity of Michıgan (Ford Nuclear Reactor) & & Ann Arbor, Mich. & $B \& W$ & Pool & 2,000 & 1957 & \\
\hline University of Utah & AGN-201-107 & Salt Lake City, Utah & AGN & Homog. solid & Neglıg & 1957 & \\
\hline Massachusetts Institute of Technology & MITR & Cambridge, Mass. & $\mathrm{ACF}$ & Heavy water & 5,000 & 1958 & \\
\hline Oregon State University & AGN-201-114 & Corvallis, Oreg. & $\mathrm{AGN}$ & Homog. solid & Neglig & 1958 & \\
\hline University of Arizona & TRIGA-Mk I & Tucson, Anz. & GGA & $U-Z r$ hydride & 100 & 1958 & \\
\hline University of Delaware & AGN-201-113 & Newark, Del & AGN & Homog. solid & Neglig. & 1958 & \\
\hline University of Oklahoma & AGN-211-102 & Norman, Okla. & AGN & Homog solid, pool & Neglig & 1958 & \\
\hline Iowa State University & UTR-10 & Ames, Iowa & ARSS & Graphite/water & 10 & 1959 & \\
\hline Leland Stanford University & & Palo Alto, Calıf & GE & Pool & 10 & 1959 & \\
\hline University of Flonda & UFTR & Gainesville, Fla. & GNEC & Graphite/water & 100 & 1959 & \\
\hline University of Wyoming & $\mathrm{L}-77$ & Laramie, Wyo & Al & Homogeneous & Neglig & 1959 & \\
\hline Virginıa Polytechnic Institute & UTR-10 & Blacksburg, Va. & ARSS & Graphite/water & 100 & 1959 & \\
\hline West Virginia University & AGN-211-103 & Morgantown, W. Va. & $A G N$ & Homog. solid, pool & Neglig. & 1959 & \\
\hline Worcester Polytechnic Institute & & Worcester, Mass. & GE & Pool & 10 & 1959 & \\
\hline Puerto Rico Nuclear Center (AEC) & L-77 & Mayaguez, $\mathrm{P}$ R & AI & Homogeneous & Neglig. & 1959 & \\
\hline Puerto Rico Nuclear Center (AEC) ${ }^{48}$ & & Mayaguez, P. R & AMF & Pool & 1,000 & 1960 & \\
\hline North Carolına State University & & Raleıgh, N. C. & Cook & Graphite/water & 10 & 1960 & \\
\hline $\begin{array}{l}\text { University of Calıfornia at Los Angeles, } \\
\text { School of Engineering and Applied Science }\end{array}$ & Educator & Los Angeles, Calif. & AMF & Graphite/water & 100 & 1960 & \\
\hline Unversity of Illmois & TRIGA-Mk III & Urbana-Champargn, Ill. & GGA & $\mathrm{U}-\mathrm{Zr}$ hydride & 1,500 & 1960 & \\
\hline Unıversity of Maryland & UMNE-1 & College Park, Md. & $\mathrm{AC}$ & Tank & 10 & 1960 & \\
\hline University of Virginia & & Charlottesville, Va. & Owner-B\&W & Pool & 1,000 & 1960 & \\
\hline University of Wisconsin 31,49 & & Madıson, Wis. & GE & Pool-TRIGA core & 1,000 & 1960 & \\
\hline $\begin{array}{l}\text { Nuclear Science Center Reactor, Texas A\&M } \\
\text { University } 31,50\end{array}$ & NSCR & College Station, Tex & Convalr & Pool-TRIGA core & 1,000 & 1961 & \\
\hline Ohı State University & & Columbus, Ohio & Lockheed & Pool & 10 & 1961 & \\
\hline
\end{tabular}


3. TEST, RESEARCH, AND UNIVERSITY REACTORS

\section{E. University Research and Teaching (Continued)}

\begin{tabular}{|c|c|c|c|c|c|c|c|}
\hline Name and/or owner & Designation & Location & $\begin{array}{l}\text { Principal } \\
\text { nuclear contractor }\end{array}$ & Type & $\begin{array}{l}\text { Power. } \\
\text { kW(t) }\end{array}$ & $\begin{array}{l}\text { Start- } \\
\text { up }\end{array}$ & $\begin{array}{l}\text { Shut- } \\
\text { down }\end{array}$ \\
\hline \multicolumn{8}{|l|}{ OPERABLE (Contınued) } \\
\hline University of Kansas & Model 4180 & Lawrence, Kans. & $\mathrm{BAC}$ & Pool & 10 & 1961 & \\
\hline University of Missoun at Rolla & & Rolla, Mo. & $\mathrm{CW}$ & Pool & 200 & 1961 & \\
\hline Unversity of Washington & Educator & Seattle, Wash. & $\mathrm{AMF}$ & Graphite/water & 100 & 1961 & \\
\hline Washington State Unuversity 31,51 & & Pullman, Wash. & GE & Pool-TRIGA core & 1,000 & 1961 & \\
\hline $\begin{array}{l}\text { Western New York Nuclear Research Center, Inc. } \\
\text { (State University of New York) }\end{array}$ & PULSTAR & Buffalo, N. Y. & $\mathrm{AMF}$ & Pool & 2,000 & 1961 & \\
\hline Cornell University $^{31}$ & TRIGA-Mk II & Ithaca, N. Y. & GGA & $\mathrm{U}-\mathrm{Zr}$ hydride & 100 & 1962 & \\
\hline Kansas State University ${ }^{31}$ & TRIGA-Mk II & Manhattan, Kans. & GGA & $\mathrm{U}-\mathrm{Zr}$ hydride & 250 & 1962 & \\
\hline Purdue University & & West Lafayette, Ind. & Lockheed & Pool & 10 & 1962 & \\
\hline Cornell University Zero Power Reactor & ZPR & Ithaca, N. Y. & Vitro & Tank & Neglig. & 1962 & \\
\hline University of Nevada & $\mathrm{L}-77$ & Reno, Nev. & $\mathrm{AI}$ & Homogeneous & Neglig. & 1963 & \\
\hline Unıversity of Texas ${ }^{31}$ & TRIGA-Mk I & Austin, Tex. & GGA & $\mathrm{U}-\mathrm{Zr}$ hydride & 250 & 1963 & \\
\hline Manhat tan College & & New York, N. Y. & AMF & Tank & Neglig. & 1964 & \\
\hline Georg1a Tech. Research Reactor & GTRR & Atlanta, Ga. & GNEC & Heavy water & 1,000 & 1964 & \\
\hline $\begin{array}{l}\text { Penn State TRIGA Reactor (Pennsylvan1a } \\
\text { State Un1versity) }\end{array}$ & PSTR & University Park, $\mathrm{Pa}$. & Owner & Pool-TRIGA core & 1,000 & 1965 & \\
\hline University of California $^{31}$ & TRIGA-Mk III & Berkeley, Calıf. & GGA & $\mathrm{U}-\mathrm{Zr}$ hydride & 1,000 & 1966 & \\
\hline University of Missour1 & & Columbia, Mo. & Owner-IC & Tank & 5,000 & 1966 & \\
\hline New York University ${ }^{53}$ & AGN-201M-105 & New York, N. Y. & $\mathrm{AGN}$ & Homog. solid & Neglig. & 1967 & \\
\hline Oregon State University ${ }^{31}$ & TRIGA-Mk II & Corvallıs, Oreg. & GGA & $\mathrm{U}-\mathrm{Zr}$ hydrıde & 1,000 & 1967 & \\
\hline Brigham Young University & $\mathrm{L}-77$ & Provo, Utah & AI & Homogeneous & Neglig. & 1967 & \\
\hline Idaho State University ${ }^{54}$ & AGN-201P-103 & Pocatello, Idaho & $\mathrm{AGN}$ & Homog. solid & Neglig. & 1967 & \\
\hline Reed College & TRIGA-Mk I & Portland, Oreg. & GGA & $\mathrm{U}-\mathrm{Zr}$ hydride & 250 & 1968 & \\
\hline Michigan State Unıversity ${ }^{30,55}$ & TRIGA-Mk I & East Lansing, Mich. & GGA & $\mathrm{U}-\mathrm{Zr}$ hydride & 250 & 1969 & \\
\hline University of California 31 & TRIGA-Mk I & Irvine, Calıf & GGA & $\mathrm{U}-\mathrm{Zr}$ hydrıde & 250 & 1969 & \\
\hline \multicolumn{8}{|l|}{ BEING BUILT } \\
\hline Mississipp1 State University ${ }^{56}$ & RRR & State College, Miss. & Owner-NSA & Homogeneous & Neglig. & & \\
\hline Lowell Technological Institute & & Lowell, Mass. & GE & Pool & 1,000 & & \\
\hline Columbia University ${ }^{31}$ & TRIGA-Mk II & New York, N Y. & GGA & $\mathrm{U}-\mathrm{Zr}$ hydride & 250 & 1970 & \\
\hline North Carolına State Unıversity & PULSTAR & Raleıgh, N. C. & $\mathrm{AMF}$ & Pool & 1,000 & 1971 & \\
\hline \multicolumn{8}{|l|}{ PLANNED } \\
\hline Colorado State University & TRIGA-Mk III & Fort Collıns, Colo. & GGA & Tank & 1,000 & & \\
\hline New York University & TRIGA-Mk I & New York, N. Y. & GGA & $\mathrm{U}-\mathrm{Zr}$ hydride & 250 & 1970 & \\
\hline New York Hall of Science 31 & TRIGA-Mk II & New York, N. Y. & GGA & $\mathrm{U}-\mathrm{Zr}$ hydride & 250 & 1971 & \\
\hline University of Calıfornıa & TRIGA-Mk I & Santa Barbara, Calıf. & GGA & $\mathrm{U}-\mathrm{Zr}$ hydride & 1,000 & & \\
\hline University of Illınois & LOPRA & Urbana, Ill. & & & 10 & & \\
\hline \multicolumn{8}{|l|}{ SHUT DOWN OR DISMANTLED } \\
\hline Willam Marsh Rice University & $A G N-211-101$ & Houston, Tex & AGN & Homog. solid, pool & Neglig. & 1959 & 1965 \\
\hline
\end{tabular}


1. MATERIALS PRODUCTION

PART II PRODUCTION REACTORS

(All owned by AEC)

\begin{tabular}{|c|c|c|c|c|c|}
\hline Designation & Nuclear designer & Type & Location & Startup & Shutdown \\
\hline \multicolumn{6}{|l|}{ OPERABLE* } \\
\hline KE Reactor & GE & Graphite & Rıchland, Wash. & 1955 & \\
\hline P Reactor & du Pont & Heavy water & Savannah River Plant, Alken, S. C. & 1954 & \\
\hline K Reactor & du Pont & Heavy water & Savannah River Plant, Aıken, S C. & 1954 & \\
\hline C Redctor & du Pont & Heavy water & Savannah River Plant, Atken, S. C. & 1955 & \\
\hline \multicolumn{6}{|l|}{ SHUT DOWN } \\
\hline R Reactor & du Pont & Heavy water & Savannah River Plant, Aıken, S. C. & 1953 & 1964 \\
\hline DR Reactor & GE & Graphite & Richland, Wash. & 1950 & 1964 \\
\hline H Reactor & $\mathrm{GE}$ & Graphite & Richland, Wash. & 1949 & 1965 \\
\hline I Reactor & du Pont & Graphite & Richland, Wash. & 1945 & 1965 \\
\hline D Reactor & du Pont & Graphite & Richland, Wash. & 1944 & 1967 \\
\hline L Reactor & du Pont & Heavy water & Savannah River Plant, Aıken, S. C. & 1954 & 1968 \\
\hline B Reactor & du Pont & Graphite & Richland, Wash. & 1944 & 1968 \\
\hline C Reactor & GE & Graphite & Richland, Wash. & 1952 & 1969 \\
\hline KW Reactor & GE & Graphite & Richland, Wash & 1955 & 1970 \\
\hline
\end{tabular}

*The N Reactor, Richland, Wash, is histed on page 12, see also footnote 11.

\section{PROCESS DEVELOPMENT}

\begin{tabular}{|c|c|c|c|c|c|c|}
\hline Name (all owned by AEC) & Designation & Location & Nuclear designer & Type & $\begin{array}{c}\text { Power, }^{1} \\
\text { kW(t) }\end{array}$ & Startup \\
\hline \multicolumn{7}{|l|}{ OPERABLE } \\
\hline Process Development P1le & PDP & Savannah River Laboratory, Aiken, S C & du Pont & Heavy water & 1 & 1953 \\
\hline Standard P1le & SP & Savannah River Laboratory, Alken, S C. & du Pont & Graphite & $2-10$ & 1953 \\
\hline Resonance Test Reactor & RTR & Savannah River Laboratory, Alken, S. C. & du Pont & Heavy water & 1 & 1967 \\
\hline Hanford 305 Test Reactor & HTR & Richland, Wash & du Pont & Graphite & Neglig. & 1944 \\
\hline Savannah River Test Pile 305 & SR-305 & Savannah River Laboratory, Aiken, S. C. & du Pont & Graphite & 1 & 1953 \\
\hline
\end{tabular}




\section{DEFENSE POWER-REACTOR APPLICATIONS}

\section{A. Remote Installations}

\section{PART III MILITARY REACTORS}

\begin{tabular}{|c|c|c|c|c|c|c|c|c|}
\hline \multirow[b]{2}{*}{ Name (all owned by DOD) } & \multirow[b]{2}{*}{ Designation $^{57}$} & \multirow[b]{2}{*}{ Location } & \multirow[b]{2}{*}{$\begin{array}{c}\text { Principal } \\
\text { nuclear contractor }\end{array}$} & \multirow[b]{2}{*}{ Type } & \multicolumn{2}{|c|}{ Power ${ }^{1}$} & \multirow[b]{2}{*}{$\begin{array}{l}\text { Start- } \\
\text { up }\end{array}$} & \multirow[b]{2}{*}{$\begin{array}{l}\text { Shut- } \\
\text { down }\end{array}$} \\
\hline & & & & & $\begin{array}{c}\text { Plant, } \\
\text { net } k W(e)\end{array}$ & $\begin{array}{l}\text { Reactor, } \\
k W(t)\end{array}$ & & \\
\hline \multicolumn{9}{|l|}{ OPERABLE } \\
\hline $\begin{array}{l}\text { Stationary Medium Power Plant No } 1 \\
\text { (formerly APPR-I) }\end{array}$ & SM-1 & Fort Belvoir, Va. & Alco & Pressunzed water & 1,855 & 10,000 & 1957 & \\
\hline Portable Medium Power Plant No 3A & PM-3A & $\begin{array}{l}\text { McMurdo Sound, } \\
\text { Antarctica }\end{array}$ & Martın & Pressurized water & 1,500 & 9,510 & 1962 & \\
\hline Statıonary Medium Power Plant No. $1 \mathrm{~A}^{58}$ & SM-1A & Fort Greely, Alaska & Alco & Pressurized water & 1,650 & 20,200 & 1962 & \\
\hline STURGIS Floatıng Nuclear Power Plant ${ }^{59}$ & $\mathbf{M H}-1 \mathbf{A}$ & Gatun Lake, Canal Zone & Martın & Pressunzed water & 10,000 & 45,000 & 1967 & \\
\hline SHUT DOWN OR DISMANTLED & & & & • & & & & \\
\hline Portable Medium Power Plant No. $2 \mathrm{~A}^{60}$ & PM-2A & Camp Century, Greenland & Alco & Pressurızed water & 1,560 & 10,000 & 1960 & 1963 \\
\hline Portable Medium Power Plant No. 1 & PM-1 & Sundance, Wyo & Martın & Pressurized water & 1,000 & 9,370 & 1962 & 1968 \\
\hline
\end{tabular}

\section{B. Propulsion (Naval)}

\begin{tabular}{|c|c|c|c|c|c|c|c|}
\hline $\begin{array}{l}\text { Name (all owned } \\
\text { by U.S. Navy) }\end{array}$ & Designation $^{61}$ & Shipbuilder & $\begin{array}{l}\text { Start- } \\
\text { up }\end{array}$ & $\begin{array}{c}\text { Name (all owned } \\
\text { by U.S. Navy) }\end{array}$ & Designation $^{61}$ & Shipbuilder & $\begin{array}{l}\text { Start- } \\
\text { up }\end{array}$ \\
\hline \multicolumn{8}{|l|}{ OPERABLE } \\
\hline USS NAUTILUS & SSN571 & Electric Boat (Groton) & 1954 & USS WHALE & SSN638 & GD (Quincy) & 1968 \\
\hline USS SEAWOLF ${ }^{2}$ & SSN575 & Electric Boat (Groton) & 1960 & USS TAUTOG & SSN639 & Ingalls & 1968 \\
\hline USS SKATE & SSN578 & Electnc Boat (Groton) & 1957 & USS BENJAMIN FRANKLIN & SSBN640 & Electric Boat (Groton) & 1965 \\
\hline USS SWORDFISH & SSN579 & Portsmouth & 1958 & USS SIMON BOLIVAR & SSBN641 & Newport News & 1965 \\
\hline USS SARGO & SSN583 & San Francisco Bay & 1958 & USS KAMEHAMEHA & SSBN642 & San Francisco Bay & 1965 \\
\hline USS SEADRAGON & SSN584 & Portsmouth & 1959 & USS GEORGE BANCROГT & SSBN643 & Electric Boat (Groton) & 1965 \\
\hline USS SKIPJACK & SSN585 & Electric Boat (Groton) & 1958 & USS LEWIS AND CLARK & SSBN644 & Newport News & 1965 \\
\hline USS HALIBUT & SSN587 & San Francisco Bay & 1959 & USS JAMES K POLK & SSBN645 & Electric Boat (Groton) & 1966 \\
\hline USS SCAMP & SSN588 & San Francisco Bay & 1961 & USS GRAYLING & SSN646 & Portsmouth & 1969 \\
\hline USS SCULPIN & SSN590 & Ingalls & 1961 & USS ASPRO & SSN648 & Ingalls & 1968 \\
\hline USS SHARK & SSN591 & Newport News & 1960 & USS WILL ROGFRS & SSBN65 & Electric Boat (Groton) & 1967 \\
\hline USS SUNFISH & SSN649 & GD (Quincy) & 1968 & USS LAPON & SSN661 & Newport News & 1967 \\
\hline USS PARGO & SSN650 & Electric Boat (Groton) & 1967 & USS GURNARD & SSN662 & San Francisco Bay & 1968 \\
\hline USS QUEENFISH & SSN651 & Newport News & 1966 & USS HAMMERHEAD & SSN663 & Newport News & 1967 \\
\hline WSS PUFFER & SSN652 & Ingalls & 1969 & USS SEA DEVIL & SSN664 & Newport News & 1968 \\
\hline S RAY & SSN653 & Newport News & 1967 & USS HAWKBILL & SSN666 & Mares Island & 1970 \\
\hline
\end{tabular}


GEORGE C MARSHALL TENR L STIMSON

USS GEORGL WASHINGTON CARVER

USS TRANCIS SCOTT KEY USS MARIANO G VALLEJO USS SNOOK

USS PERMIT

USS PLUNGER

USS BARB

USS TULLIBEE

USS GEORGE WASHINGTON

USS PATRICK HENRY

USS THEODORE ROOSEVELT

USS ROBERT E LEE

USS ABRAHAM LINCOLN

USS POLLACK

USS HADDO

USS JACK

USS TINOSA

USS DACE

USS ETHAN ALLEN

USS SAM HOUSTON

USS THOMAS A EDISON

USS JOHN MARSHALL

USS GUARDHISH

USS FLASHER

USS GREENLING

USS GATO

USS LAFAYETTE

USS THOMAS JEFFERSON

USS ANDREW JACKSON

USS JOHN ADAMS

USS HADDOCK

USS JAMES MONROE

USS NATHAN HALE

USS WOODROW WILSON

USS HENRY CLAY

USS DANIEL WEBSTER

USS JAMES MADISON

USS TECUMSEH

USS DANIEL BOONE

USS JOHN C. CALHOUN

USS ULYSSES S GRANT

USS VON STEUBEN

USS CASIMIR PULASKI

USS STONLWALL JACKSON

USS SAM RAYBURN

USS NATHANAEL GREENE

USS STURGEON
SSBN 654

SSBN655

SSBN656

SSBN657

SSBN658

SSN 592

SSN594

SSN595

SSN596

SSN597

SSBN600

SSBN601

SSBN602

SSN603

SSN604

SSN605

SSN606

SSN607

SSBN608

SSBN609

SSBN610

SSBN611

SSN612

SSN613

SSN614

SSN615

SSBN618

SSBN619

SSBN620

SSN621

SSBN622

SSBN623

SSBN624

SSBN625

SSBN626

SSBN627

SSBN628

SSBN629

SSBN630

SSBN631

SSBN632

SSBN633

SSBN634

SSBN635

SSBN636

SSN637
Boat (Groton) 1966

Newport News

1966

Electric Boat (Groton) 1966

Ingalls

San Francisco Bay

Ingalls

Electric Boat (Groton) 1960

Electric Boat (Groton) 1959

Electric Boat (Groton) 1960

San Francisco Bay $\quad 1960$

Newport News 1960

Portsmouth 1960

NYSC

NYSC

Portsmouth

Portsmouth

Ingalls

Boat (Groton)

GD (Quincy)

GD (Quincy)

1963

1963

Newport News

San $\mathrm{H}$ rancisco Bay 1963

Portsmouth

Ingalls

Newport News

1963

San Francisco Bay 1963

Newport News

Electnc Boat (Groton)

Newport News

1964

San Francisco Bay

Newport News

1964

(1964

Newport News

Portsmouth

Electric Boat (Groton)

1964

964

1966
USS BERGALL

USS SPADEFISH

USS SEA HORSE

USS FINBACK

USS NARWHAL

USS FLYING FISH

USS TREPANG

USS LONG BEACH (2 reactors)

USS ENTERPRISE ( 8 reactors)

USS BAINBRIDGE ( 2 reactors)

USS TRUXTUN ( 2 reactors)

Deep Submergence Research

Vehricle

BEING BUILT

POGY

SAND LANCE

GUITARRO

PINTADO

BLUEFISH

BILLFISH

DRUM

ARCHERFISH

SILVERSIDES

REDFISH

BATFISH

TUNNY

PARCHE

CAVALLA

Submarne

Submarine

Submarne

NIMITZ ( 2 reactors)

EISENHOWER

(2 reactors)

CALITORNIA

(2 reactors)

Guided Missle Frigate

( 2 reactors)

\section{PLANNED}

Submarine

Submanne

Submanne

Guided Missile Frigate

( 2 reactors)

SHUT DOWN OR DISMANTLED

SEAWOLF Sodium Reactor ${ }^{62}$

USS THRESHER ${ }^{63}$

USS SCORPION ${ }^{63}$
SSN667

SSN668

SSN669

SSN670

SSN671

SSN673

SSN674

CGN9

CVAN65

DLGN25

DLGN35

NR-1

SSN647

SSN660

SSN665

SSN672

SSN675

SSN676

SSN677

SSN678

SN679

SSN680

SSN681

SSN682

SSN683

SSN684

SSN685

SSN686

SSN687

CVAN68

CVAN69

DLGN36

DLGN37

Boat (Groton)

Newport News

Newport News 1969

Electric Boat (Groton) 1969

Electric Boat (Groton) 1969

Electric Boat (Groton) 1970

Bethlehem

1960

1962

Electric Boat (Groton) 1969

NYSC/Ingalls

Portsmouth

Mares Island

Mares Island

Electric Boat (Groton)

Electric Boat (Groton)

Mares Island

Electric Boat (Groton)

Electric Boat (Groton)

Ingalls

Electric Boat (Groton)

Ingalls

Electric Boat (Groton)

Electric Boat (Groton)

Newport News

Newport News

Newport News

Newport News

Newport News

SSN688 Unassigned

Unassigned

SSN690 Unassigned

DLGN38 Unassigned

SSN593

SSN586

\begin{tabular}{|c|c|}
\hline & $\begin{array}{l}\text { Start- } \\
\text { up }\end{array}$ \\
\hline Electric Boat (Groton) & $\overline{1956}$ \\
\hline Portsmouth & 1961 \\
\hline Electric Boat (Groton) & 1960 \\
\hline Electne Boat (Groton) & 1959 \\
\hline
\end{tabular}


A. Electric-Power Experiments and Prototypes

\begin{tabular}{|c|c|c|c|c|c|c|c|c|}
\hline \multirow[b]{2}{*}{ Name (all owned by AEC) } & \multirow[b]{2}{*}{ Designation $^{57}$} & \multirow[b]{2}{*}{ Location } & \multirow{2}{*}{$\begin{array}{c}\text { Principal } \\
\text { nuclear } \\
\text { contractor }\end{array}$} & \multirow[b]{2}{*}{ Type } & \multicolumn{2}{|c|}{ Power $^{1}$} & \multirow[b]{2}{*}{$\begin{array}{l}\text { Start- } \\
\text { up }\end{array}$} & \multirow[b]{2}{*}{$\begin{array}{l}\text { Shut } \\
\text { down }\end{array}$} \\
\hline & & & & & $\begin{array}{c}\text { Plant, } \\
\text { net kW(e) }\end{array}$ & $\begin{array}{l}\text { Reactor, } \\
k W(t)\end{array}$ & & \\
\hline \multicolumn{9}{|l|}{ SHUTDOWN OR DISMANTLED } \\
\hline Stationary Low Power Plant No 1 & SL-1 & NRTS, Idaho & ANL & Bolling water & 300 & 2,200 & 1958 & 1961 \\
\hline Gas Cooled Reactor Experment & GCRE & NRTS, Idaho & AGN & $\begin{array}{l}\text { Gas cooled, light } \\
\text { water moderated }\end{array}$ & No elec & 2,200 & 1960 & 1962 \\
\hline Mobile Low Power Plant No 1 & ML-1 & NRTS, Idaho & $\mathrm{AGN}$ & $\begin{array}{l}\text { Gas cooled, light } \\
\text { water moderated }\end{array}$ & 300 & 3,300 & 1961 & 1965 \\
\hline
\end{tabular}

\section{B. Propulsion Experiments and Prototypes}

$\tilde{\Sigma}$

\begin{tabular}{|c|c|c|c|c|c|c|c|}
\hline Name (all owned by AEC) & Designation & Location & $\begin{array}{l}\text { Principal } \\
\text { nuclear } \\
\text { contractor }\end{array}$ & Type & $\begin{array}{c}\text { Power, }^{1} \\
\text { kW(t) }\end{array}$ & $\begin{array}{l}\text { Start- } \\
\text { up }\end{array}$ & $\begin{array}{l}\text { Shut- } \\
\text { down }\end{array}$ \\
\hline \multicolumn{8}{|l|}{ OPERABLE } \\
\hline S1W Reactor Facılity & $\mathrm{S} 1 \mathrm{~W}$ & NRTS, Idaho & West & Pressurnzed water & & 1953 & \\
\hline Large Ship Reactor Prototype (2 reactors) & $\mathrm{A} 1 \mathrm{~W}$ & NRTS, Idaho & West. & Pressurized water & & 1958 & \\
\hline Submarıne Advanced Reactor Prototype & S3G & West Milton, N Y & GE & Pressurized water & & 1958 & \\
\hline Small Submanne Reactor Prototype & S1C & Windsor, Conn & Comb & Pressurnzed water & & 1959 & \\
\hline Destroyer Reactor Prototype & D $1 G$ & West Milton, N Y & GE & Pressurnzed water & & 1962 & \\
\hline Natural Circulation Test Plant & S5G & NRTS, Idaho & $\mathrm{GE}$ & Pressurized water & & 1965 & \\
\hline \multicolumn{8}{|l|}{ SHUT DOWN OR DISMANTLED } \\
\hline Submarıne Intermedıate Reactor Mark A & $\mathrm{S} 1 \mathrm{G}$ & West Milton, N Y & GE & Sodium & & 1955 & 1957 \\
\hline Aircraft Reactor Experiment & ARE & Oak Ridge, Tenn & ORNL & Molten salt & 1,500 & 1954 & 1954 \\
\hline Heat Transfer Reactor Expenment No 1 & HTRE-1 & NRTS, Idaho & ANPD & Air cooled & 20,000 & 1956 & 1957 \\
\hline Heat Transfer Reactor Expenment No 2 & HTRE-2 & NRTS, Idaho & ANPD & Air cooled & 14,000 & 1957 & 1961 \\
\hline Heat Transfer Reactor Experiment No 3 & HTRE-3 & NRTS, Idaho & ANPD & Air cooled & 32,000 & 1958 & 1961 \\
\hline Experumental Propulsion Test Reactor & TORY IIA & NTS, Nev & UCLRL & Aur cooled & 150,000 & 1960 & 1961 \\
\hline Experimental Propulsion Test Reactor ${ }^{64}$ & TORY IIC & NTS, Nev & UCLRL & Air cooled & 600,000 & 1964 & 1964 \\
\hline
\end{tabular}




\section{TEST AND RESEARCH}

\section{A. Test}

\begin{tabular}{|c|c|c|c|c|c|c|c|}
\hline $\begin{array}{l}\text { Name (all owned by AEC } \\
\text { except as noted) }\end{array}$ & Desıgnation & Location & $\begin{array}{c}\text { Principal } \\
\text { nuclear } \\
\text { contractor }\end{array}$ & Type & $\begin{array}{c}\text { Power, } \\
\text { kW(t) }\end{array}$ & $\begin{array}{l}\text { Start- } \\
\text { up }\end{array}$ & $\begin{array}{l}\text { Shut- } \\
\text { down }\end{array}$ \\
\hline \multicolumn{8}{|l|}{ OPERABLE } \\
\hline Ground Test Reactor (USAF) & GTR & Fort Worth, Tex. & Convalr & Pool & 10,000 & 1953 & \\
\hline Aerospace Systems Test Reactor (USAF) & ASTR & Fort Worth, Tex. & Convanr & Light water & 10,000 & 1954 & \\
\hline Tower Sheldıng Reactor No. 2 & TSR-2 & Oak Ridge, Tenn. & ORNL & Light water & 100 & 1960 & \\
\hline \multicolumn{8}{|l|}{ SHUT DOWN OR DISMANTLED } \\
\hline Tower Shieldıng Reactor No. 1 & TSR-1 & Oak Ridge, Tenn. & ORNL & Tank & 500 & 1954 & 1958 \\
\hline Nuclear Engineering Test Reactor (USAF) & NETR & Dayton, Ohio & Maxon-AC & Tank & 10,000 & 1965 & 1970 \\
\hline
\end{tabular}

\section{B. Research}

\begin{tabular}{|c|c|c|c|c|c|c|c|}
\hline Name and/or owner & Designation & Location & $\begin{array}{c}\text { Principal } \\
\text { nuclear } \\
\text { contractor }\end{array}$ & Type & $\begin{array}{c}\text { Power, } \\
k W(t)\end{array}$ & $\begin{array}{l}\text { Start- } \\
\text { up }\end{array}$ & $\begin{array}{l}\text { Shut- } \\
\text { down }\end{array}$ \\
\hline \multicolumn{8}{|l|}{ OPERABLE } \\
\hline Thermal Test Reactor No 1 (AEC) & TTR-1 & Schenectady, N. Y & KAPL & Graphite & 10 & 1951 & \\
\hline $\begin{array}{l}\text { Diamond Ordnance Radiation Facility } \\
\text { (Harry Diamond Laboratones, USA) }^{31}\end{array}$ & DORF & Forest Glen, Md. & GGA & TRIGA-Mk F & 250 & 1961 & \\
\hline $\begin{array}{l}\text { Armed Forces Radiobiology Research } \\
\text { Institute (DASA, DOD) }\end{array}$ & AFRRI & Bethesda, Md. & GGA & $\mathrm{U}-\mathrm{Zr}$ hydride & 100 & 1962 & \\
\hline $\begin{array}{l}\text { Walter Reed Research Reactor (Walter Reed } \\
\text { Army Institute of Research, USA) }\end{array}$ & WRRR & Washıngton, D. C. & AI & Homogeneous & 50 & 1962 & \\
\hline $\begin{array}{l}\text { Fast Burst Reactor Facility (Army Missile } \\
\text { Test and Evaluation Directorate, USA) }\end{array}$ & ГBRF & White Sands, N. Mex. & Kaman & $\begin{array}{l}\text { Bare, fast, } \\
\text { prompt burst }\end{array}$ & 10 & 1964 & \\
\hline Nuclear Effects Reactor (AEC) & Super KUKLA & NTS, Nev. & UCLRL & Prompt burst & Transient & 1964 & \\
\hline $\begin{array}{l}\text { Aberdeen Pulsed Reactor Facility (Ballistic } \\
\text { Research Laboratories, USA) }\end{array}$ & APRF & Aberdeen, Md. & UNC & $\begin{array}{l}\text { Bare, fast, } \\
\text { prompt burst }\end{array}$ & 10 & 1968 & \\
\hline \multicolumn{8}{|l|}{ SHUT DOWN OR DISMANTLED } \\
\hline Naval Research Reactor (USN) ${ }^{3}$ & NRR & Washıngton, D. C & NRL & Pool & 1,000 & 1956 & 1970 \\
\hline U. S. Naval Postgraduate School (USN) ${ }^{3}$ & AGN-201-100 & Monterey, Calıf. & AGN & Homog. solid & Neglig. & 1956 & 1970 \\
\hline $\begin{array}{l}\text { Army Materials Research Reactor (Army } \\
\text { Materials and Mechanıcs Research Center, } \\
\text { USA) }\end{array}$ & AMRR & Watertown, Mass. & $\mathrm{BAC}$ & Pool & 5,000 & 1960 & 1970 \\
\hline
\end{tabular}




\section{POWER REACTORS ${ }^{65}$}

PART IV REACTORS FOR EXPORT

\section{A. Central-Station Electric Power}

\begin{tabular}{|c|c|c|c|c|c|c|}
\hline \multirow[b]{2}{*}{ Name and/or owner } & \multirow[b]{2}{*}{ Locatıon } & \multirow{2}{*}{$\begin{array}{l}\text { Principal } \\
\text { nuclear } \\
\text { contractor }\end{array}$} & \multirow[b]{2}{*}{ Type } & \multicolumn{2}{|c|}{ Power ${ }^{1}$} & \multirow[b]{2}{*}{$\begin{array}{l}\text { Start- } \\
\text { up }\end{array}$} \\
\hline & & & & $\begin{array}{l}\text { Plant, } \\
\text { net } k W(e)\end{array}$ & $\begin{array}{l}\text { Reactor, } \\
k W(t)\end{array}$ & \\
\hline \multicolumn{7}{|l|}{ OPERABLE } \\
\hline Germany, Kahl Nuclear Power Statıon (Rhıne-Westphalıa Power Co, RWE) & Kahl-am-Main & GE & Bollıng water & 15,600 & 60,000 & 1960 \\
\hline Italy, Garigliano Nuclear Power Statıon (Project ENSI of SENN) & $\begin{array}{l}\text { Punta Fiume (on } \\
\text { Garigliano River) }\end{array}$ & GE & Bolling water & 150,000 & 506,000 & 1963 \\
\hline Japan, Japan Power Demonstration Reactor (JAERI) & Toka1-Mura & GE & Bolling water & 11,250 & 45,000 & 1963 \\
\hline Italy, Project Enrico Fermı of SELNI, Edisonvolta & Trıno Vercellese & West & Pressurized water & 256,000 & 615,000 & 1964 \\
\hline France, Franco-Belgian Society for Nuclear Energy of Ardennes, SENA & Givet (near Chooz) & West & Pressurned water & 266,000 & 825,000 & 1966 \\
\hline Germany, Kernkraftwerk-KWE-Bayernwerk, or KRB & $\begin{array}{l}\text { Gundremmingen } \\
\text { (near Gunzburg) }\end{array}$ & GE & Bolling water & 237,000 & 801,000 & 1966 \\
\hline Spaın, José Cabrera Nuclear Power Plant, Unıt 1 & Near Madrid & West & Pressunzed water & 153,000 & 515,000 & 1968 \\
\hline India, Tarapur Nuclear Power Station, Units 1 and 2 & $\begin{array}{l}\text { Tarapur (north of } \\
\text { Bombay) }\end{array}$ & $\mathrm{GE}$ & Bollng water & 380,000 & $1,322,000$ & 1969 \\
\hline $\begin{array}{l}\text { Japan, Tsuruga Nuclear Power Plant (Japan Atomic Power Company, } \\
\text { JAPCO No 2) }\end{array}$ & Tsuruga, Honshu & GE & Boulung water & 432,800 & & 1969 \\
\hline Swıtzerland, NOK Nuclear Electric Generatıng Statıon, Unıt 1 & $\begin{array}{l}\text { Beznau (near } \\
\text { Baden) }\end{array}$ & West & Pressurnzed water & 350,000 & $1,130,000$ & 1969 \\
\hline \multicolumn{7}{|l|}{ BEING BUILT } \\
\hline Japan, Fukushıma Station, Unit 1 (Tokyo Electric Power Corp ) & Fatuba, Honshu & GE & Boiling water & 440,000 & & 1970 \\
\hline Japan, Fukushıma Station, Unit 2 (Tokyo Electric Power Corp) & Fatuba, Honshu & $\mathrm{GE}$ & Bolling water & 784,000 & & 1973 \\
\hline Japan, Mhama Nuclear Power Station, Unit 1 (Kansai Electric Power Co ) & Niu, Honshu & West & Pressurzed water & 340,790 & & 1970 \\
\hline Japan, Mihama Nuclear Power Station, Unit 2 (Kansa Electric Power Co ) & Niu, Honshu & West & Pressurzed water & 500,000 & & 1972 \\
\hline Spain, José Cabrera Nuclear Power Plant, Unit 2 & Near Madrid & West & Pressurnzed water & 450,000 & & 1972 \\
\hline Spaun, Santa Marna de Garona Nuclear Power Plant & Near Bilbao & GE & Bolling water & 440,000 & & 1969 \\
\hline Switzerland, NOK Nuclear Electric Generating Station, Unit 2 & Beznau (near Baden) & West & Pressunzed water & 350,000 & $1,130,000$ & 1972 \\
\hline Switzerland, Bermische Kraftwerk A G, or BKW & Muehleberg (near Bern) & GE & Bolling water & 306,200 & & 1971 \\
\hline \multicolumn{7}{|l|}{ PLANNED } \\
\hline Sweden, Swedish State Power Board & Ringhals & West. & Pressurized water & 809,000 & & 1974 \\
\hline Japan, Kansa1 Electric Power Co, Unit 3 & Takahama & West & Pressurzed water & 826,000 & & 1974 \\
\hline Korea, Korea Electnc Power Co, Unit 1 & & West & Pressurnzed water & 500,000 & & 1975 \\
\hline Talwan, Talwan Power Co & Chm Shan & GE & Bolling water & 604,000 & & 1975 \\
\hline Italy & Pizcenza/Cremona & GE & Bolling water & 750,000 & & 1975 \\
\hline
\end{tabular}




\begin{tabular}{cccccc}
\multicolumn{1}{c}{ Name } & Owner & Desıgner & Designation & Type & $\begin{array}{c}\text { Start } \\
\text { up }\end{array}$ \\
\hline $\begin{array}{c}\text { OPERABLE } \\
\text { SSW for HMS DREADNOUGHT }\end{array}$ & Great Britain & West & S5W & Pressurzed water & 1962 \\
\hline
\end{tabular}

\section{TEST, RESEARCH, AND TEACHING}

\section{A. General Irradiation Test}

\begin{tabular}{|c|c|c|c|c|c|}
\hline Owner & Location & $\begin{array}{c}\text { Principal } \\
\text { nuclear } \\
\text { contractor }\end{array}$ & Type & $\begin{array}{c}\text { Power, }^{1} \\
\text { kW(t) }\end{array}$ & $\begin{array}{l}\text { Start- } \\
\text { up }\end{array}$ \\
\hline \multicolumn{6}{|l|}{ OPERABLE } \\
\hline Japan, Atomic Energy Research Instıtute & Tokal-Mura & AMF & Heavy water, tank & 10,000 & 1960 \\
\hline Netherlands, Reactor Center & Petten & $\mathrm{AC}$ & Tank (MTR) & 45,000 & 1961 \\
\hline South Afnca, Atomic Energy Board & $\begin{array}{l}\text { Pelındaba (near } \\
\text { Pretoria) }\end{array}$ & $\mathrm{AC}$ & Tank & 20,000 & 1965 \\
\hline Sweden, A tomic Energy Company & Studsvik & $\mathrm{AC}$ & Tank (MTR) & 30,000 & 1960 \\
\hline
\end{tabular}

\section{B. General Research}

\section{OPERABLE}

Australıa, A tomic Energy Commission

Austria, Seibersdorf Research Center

Colombia, Colombian Institute of Nuclear Affars

Denmark, Atomic Energy Commission

Denmark, Atomic Energy Commission

Germany, Society for the Utılization of Nuclear

Energy in Shipbulding and Navigation, Inc

Germany, Brown Boven/Krupp ${ }^{66}$

West Berlın, City of (Instıtute for Nuclear Research)

Greece, Atomic Energy Commission

Indonesia, Institute for Atomic Energy

Israel, A tomic Energy Commission

Italy, Center for Military Applications of Nuclear Energy

Italy, Natıonal Committee for Nuclear Energy

Italy, National Committee for Nuclear Energy

Italy, SORIN Nuclear Center

Korea, A tomic Energy Commission

Pakıstan, Atomıc Energy Commıssıon

Lucas Heights,
New South Wales
Setbersdorf
Bogota
Riso
Riso
Geesthacht
Julıch
West Berlın
Athens
Bandung
Nahal Soreq
Near Pisa
Ispra
Rome
Saluggia
Seoul
Islamabad

ARSS
AMF
Lockheed
AI
FW
B\&W
Interatom
AI
AMF
GGA
AMF
B\&W
AC
GGA
AMF
GGA
AMF

UTR 10
Pool
Pool
L-55
Tank
Pool
L-77A
L-54
Pool
TRIGA-Mk II
Pool
Pool
Heavy water, tank
TRIGA-Mk II
Pool
TRIGA-Mk II
Pool

$\begin{array}{ll}10 & 1961 \\ & \\ 5,000 & 1960 \\ 10 & 1965 \\ 05 & 1957 \\ 5,000 & 1958 \\ 5,000 & 1958 \\ & \\ 001 & 1967 \\ 50 & 1958 \\ 1,000 & 1961 \\ 250 & 1964 \\ 5,000 & 1960 \\ 5,000 & 1963 \\ 5,000 & 1959 \\ 1,000 & 1960 \\ 1,000 & 1959 \\ 100 & 1962 \\ 5,000 & 1965\end{array}$


B. General Research (Continued)

\begin{tabular}{|c|c|c|c|c|c|c|}
\hline Owner & Location & $\begin{array}{l}\text { Principal } \\
\text { nuclear } \\
\text { contractor }\end{array}$ & Type & $\begin{array}{c}\text { Power, }^{1} \\
\text { kW(t) }\end{array}$ & $\begin{array}{c}\text { Start } \\
\text { up }\end{array}$ & $\begin{array}{l}\text { Shut } \\
\text { down }\end{array}$ \\
\hline \multicolumn{7}{|l|}{ OPERABLE (Contınued) } \\
\hline Philippınes, Natıonal Science Development Board & Quezon City & GE & Pool & 1,000 & 1963 & \\
\hline Portugal, Nuclear Energy Board & Sacavém & AMF & Pool & 1,000 & 1961 & \\
\hline Spain, Nuclear Energy Board & Moncloa & GE & Pool & 3,000 & 1958 & \\
\hline Switzerland, Reactor, Inc 67 & Wuerenlingen & ORNL & Pool & 1,000 & 1955 & \\
\hline Turkey, A tomic Energy Commission & Istanbul & $\mathrm{AMF}$ & Pool & 1,000 & 1962 & \\
\hline Vietnam, Institute of Nuclear Research & Dalat & GGA & TRIGA-Mk II & 250 & 1963 & \\
\hline Venezuela, Institute for Scientific Research & Caracas & $\mathrm{GE}$ & Pool & 3,000 & 1960 & \\
\hline Yugoslavia, Josef Stefan Nuclear Institute ${ }^{31}$ & Podganca & GGA & TRIGA Mk II & 250 & 1966 & \\
\hline Mexico, National Commıssion for Nuclear Energy ${ }^{31}$ & Salazar & GGR & TRIGA-Mk III & 1,000 & 1968 & \\
\hline \multicolumn{7}{|l|}{ BEING BUILT } \\
\hline Italy, Natıonal Committee for Nuclear Energy & Padua & AMF & Pool & Neglig & 1970 & \\
\hline Korea, Atomic Energy Research Institute & Seoul & GGA & TRIGA-Mk III & 2,000 & 1974 & \\
\hline \multicolumn{7}{|l|}{ SHUT DOWN OR DISMANTLED } \\
\hline Japan, A tomic Energy Research Institute & Tokar-Mura & AI & L-54 & 50 & 1957 & 1969 \\
\hline
\end{tabular}

\section{University Research and Teaching}

\begin{tabular}{|c|c|c|c|c|c|}
\hline Owner & Location & $\begin{array}{l}\text { Principal } \\
\text { nuclear } \\
\text { contractor }\end{array}$ & Type & $\begin{array}{c}\text { Power, } \\
\text { kW(t) }\end{array}$ & $\begin{array}{c}\text { Start- } \\
\text { up }\end{array}$ \\
\hline \multicolumn{6}{|l|}{ OPERABLE } \\
\hline Austrıa, Vienna Polytechnic Instıtute ${ }^{31}$ & Vienna & GGA & TRIGA-Mk II & 250 & 1962 \\
\hline Brazıl, Unıversıty of São Paulo & São Paulo & $\mathrm{B} \& \mathrm{~W}$ & Pool & 5,000 & 1957 \\
\hline Brazıl, University of Minas Gera1s ${ }^{68}$ & Belo Horizonte & GGA & TRIGA-Mk I & 30 & 1960 \\
\hline Canada, McMaster Unıversity & Hamilton, Ont & AMF & Pool & 1,000 & 1959 \\
\hline China, Republic of (National Tsing-Hua University) & Hsinchu & GE & Pool & 1,000 & 1961 \\
\hline Congo, Republic of the, (University of Lovanium) ${ }^{69}$ & Kinshasa & GGA & TRIGA-Mk I & 50 & 1959 \\
\hline Finland, Institute of Technology 31 & Helsınkı & GGA & TRIGA-Mk II & 250 & 1962 \\
\hline Germany, Technical Unıversity of Munıch & Munich & AMF & Pool & 2,500 & 1957 \\
\hline Germany, Universities of Frankfurt and Darmstadt & Frankfurt & AI & L-54 & 50 & 1958 \\
\hline Germany, Johannes Gutenberg University of Mainz ${ }^{31}$ & Mainz & GGA & TRIGA-Mk II & 100 & 1965 \\
\hline Germany, Institute for Nuclear Medicine ${ }^{70}$ & Heidelkerg & GGA & TRIGA-Mk & 250 & 1966 \\
\hline Iran, University of Tehran & Tehran & $\mathrm{AMF}$ & Pool & 5,000 & 1967 \\
\hline Italy, University of Milan & Milan & AI & $\mathrm{L}-54$ & 50 & 1959 \\
\hline Italy, University of Palermo & Palermo & AGN & $201-110$ & Neglig & 1960 \\
\hline Italy, University of Pavia ${ }^{30}$ & Pavia & GGA & TRIGA-Mk II & 250 & 1965 \\
\hline apan, Kınkı Unıversıty & Osaka & ARSS & UTR-10 & Neglig & 1961 \\
\hline
\end{tabular}


Netherlands, Delft Technical University ${ }^{71}$

Yokosuka

Switzerland, University of Basel ${ }^{72}$

Switzerland, University of Geneva ${ }^{73}$

Basel

AMF

Thalland, Chulalongkorn Unive

Geneva

AGN

Thalland, Chulalongkorn University

AGN

United Kıngdom, Scottısh Research Reactor Center

London

CW

Uruguay, University of Montevideo ${ }^{74}$

East Kılbride

ARSS

PLANNED

Germany, Association for Radiation Research ${ }^{30,70}$

Montevideo

ARSS

TRIGA-Mk II

\section{IDENTIFICATION OF FACILITIES}

\section{PART V CRITICAL ASSEMBLY FACILITIES}

\begin{tabular}{|c|c|c|c|c|}
\hline Abbreviation & Name and location of facility & Operator & $\begin{array}{l}\text { No. of } \\
\text { cells }\end{array}$ & $\begin{array}{l}\text { No. of } \\
\text { control panels }\end{array}$ \\
\hline ANL & Argonne National Laboratory (AEC), Argonne, Ill. & ANL & 4 & 4 \\
\hline ANL-ID & Argonne National Laboratory, Idaho Division (AEC), NRTS, Idaho & ANL & 2 & 2 \\
\hline ARMF-I and -II & Advanced Reactivity Measurement Facility (AEC), NRTS, Idaho & INC & 2 & 2 \\
\hline ATF & SNAP Acceptance Test Fac1lity (AEC), Santa Susana, Calıf. & AI & 1 & 1 \\
\hline ATRC & Advanced Test Reactor Critical Facility (AEC), NRTS, Idaho & INC & 1 & 1 \\
\hline Bettis & Bettis Atomic Power Laboratory (AEC), Pittsburgh, Pa. & West. & 6 & 6 \\
\hline $\mathrm{B} \& \mathrm{~W}$ & The Babcock \& Wilcox Co., Lynchburg, Va. ${ }^{3}$ & Owner & 3 & 3 \\
\hline Comb. & $\begin{array}{l}\text { Nuclear Engne Laboratory of Combustion Engineenng, Inc., } \\
\text { Windsor, Conn. }\end{array}$ & Owner & 3 & 2 \\
\hline ECEL & $\begin{array}{l}\text { Epithermal Critical Experiment Laboratory, Santa Susana, } \\
\text { Calif. }\end{array}$ & AI & 1 & 1 \\
\hline ETRC & Engineermg Test Reactor Critıcal Facility (AEC), NRTS, Idaho & INC & 1 & 1 \\
\hline GGA & Gulf General Atomic Incorporated, San Diego, Calif. ${ }^{3}$ & Owner & 2 & 2 \\
\hline KAPL & Knolls A tomic Power Laboratory (AEC), Schenectady, N. Y. & GE & 6 & 6 \\
\hline LASL & Los Alamos Screntific Laboratory (AEC), Los Alamos, N. Mex & LASL & 3 & 3 \\
\hline Lockheed & $\begin{array}{l}\text { Lockheed Aircraft Co., Critical Facility for RER, } \\
\text { Dawsonville, Ga. }{ }^{3}\end{array}$ & Owner & 1 & 1 \\
\hline LPTF & Low Power Test Facilıty (AEC), NRTS, Idaho & GE & 2 & 2 \\
\hline Martın & Martın Co., Middle River, $\mathrm{Md}^{3}$ & Owner & 3 & 3 \\
\hline NASA & Lewis Research Center, Cleveland, $\mathrm{Oh}_{10}^{3}$ & Owner & 3 & 2 \\
\hline OR-CEF & Oak Rıdge Critıcal Experıment Facılıty (AEC), Oak Rıdge, Tenn. & UCC-ND & 3 & 3 \\
\hline ORNL-PCA & Pool Critical Assembly, BSF Pool (AEC), Oak Ridge, Tenn. & ORNL & 1 & 1 \\
\hline PNL-CML & Critical Mass Laboratory (AEC), Richland, Wash. & PNL & 1 & 1 \\
\hline PNL-PRCF & Plutonium Recycle Critical Facility (AEC), Richland, Wash. & PNL & 1 & 1 \\
\hline RFP-NSF & Nuclear Safety Facilıty, Rocky Flats Plant (AEC), Colo. & DOW & 1 & 1 \\
\hline Rensselaer & Rensselaer Polytechnic Institute, Troy, N. Y. ${ }^{3}$ & Owner & 1 & 1 \\
\hline
\end{tabular}


1. IDENTIFICATION OF FACILITIES (Continued)

PART V CRITICAL ASSEMBLY FACILITIES

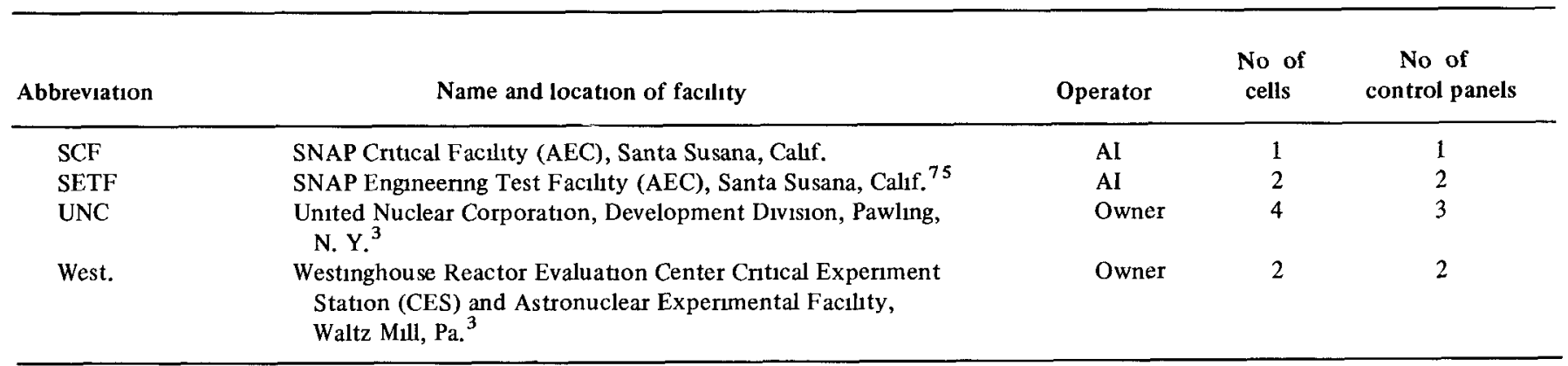

\section{IDENTIFICATION OF EXPERIMENTS AND STUDIES}

\section{A. Civilian}

\begin{tabular}{|c|c|c|c|}
\hline Facility & Subject of current experument or study & Designation & Startup \\
\hline \multicolumn{4}{|l|}{ OPERABLE } \\
\hline AI, ECEL & Nuclear properties of fast reactor systems & ECEL & 1960 \\
\hline AI, SCF & NASA Fast Reactor Critical Assembly & SCA-5 & 1970 \\
\hline $\mathrm{AI}, \mathrm{SCF}$ & SNAP Safety Program & SCA-4A and $4 \mathrm{~B}$ & 1962 \\
\hline ANL & Basic fast reactor studies and mock-up for LMFBR & ZPR-6 & 1963 \\
\hline $\mathrm{ANL}^{76}$ & Vacant & ZPR-7 & 1956 \\
\hline ANL & Vacant & ZPR-5 & 1957 \\
\hline ANL & Basic fast reactor studies and mock-up for LMFBR & ZPR-9 & 1967 \\
\hline ANL-ID & Basic fast reactor studies and mock-up for LMFBR & ZPR-3 & 1955 \\
\hline ANL-ID & Basic fast reactor studies and mock-up for LMFBR & ZPPR & 1969 \\
\hline $\begin{array}{l}\text { ARMF-II, Test Reactor Area } \\
\text { (INC) }\end{array}$ & $\begin{array}{l}\text { Reactor-physics constants for thermal and fast } \\
\text { reactors, studies of fast-zoned reactor cores }\end{array}$ & ARMF-II & 1962 \\
\hline $\begin{array}{l}\text { ARMF-I, Test Reactor Area } \\
\text { (INC) }\end{array}$ & $\begin{array}{l}\text { Reactor-physics constants and reactivity changes caused } \\
\text { by test-reactor irradiation }\end{array}$ & ARMF-I & 1960 \\
\hline ATRC, Test Reactor Area (INC) & ATR physics, core-loading and core-design measurements & ATRC & 1964 \\
\hline Bettıs & Critical experiments & CCFA & 1954 \\
\hline Bettis & LWB physics 77 & LWBCC & 1963 \\
\hline
\end{tabular}


B\&W, Cell 1

B\&W, Cell $2^{78}$

$B \& W$ Cell $3^{78}$

ETRC, Test Reactor Area (INC)

GGA

GGA

LASL, Kiva I

LASL, Kıva III

LPTT, Cell 1

LPTF, Cell 2

NASA, Materials and

Stresses Bullding ${ }^{79}$

NASA, Materials and

Stresses Bu1lding

OR-CEF, Bu1lding 9213

Cell E

OR-CEF, Building 9213 ,

Cell S

Advanced Test Reactor critical

1962

Spectral Shuft Control Reactor

Small-lattıce experıment

ETR physics, core-loading and core-design measurements

ETRC

Accelerator Pulsed Fast Critical Assembly

Core design of thermionic power reactors

Cold criticals for Rover reactors

Cold critical operation of Rover test reactors,

environmental chamber, and general-purpose

critical assembly

NASA Sphencal Cavity Reactor Cntıcal Experiment

1958

THRITS experiments

NASA Test Reactor cntical expenments (NASA-ZPR-I system)

NASA Modified Zero Power Reactor II, solution-type

critical facility

Subcritical measurements in metallic uranium and

Plexiglass lattices

Uranium Metal-Graphite Array

APFA-III

1970

ZEPO and Honeycomb 1957

Godiva-IV

Reactor-physics and nuclear-safety studies with homogeneous uranium solutions, slightly enriched-uranium

lattices in water, investigations of neutron absorbers

as poisons in chemical-processing equipment, HFIR

core reactivity studies

ORNL-PCA, Buldıng 3010

Physics research on reactivity effects

\section{3}

1966

1959

1969

1950

1950

1950

PNL-CML

Plutonium criticals

Plutonium recycle criticals

Critical facility for flight-system acceptance testing

Critical expenment assembly

Rensselaer

Proof Test Facility

Reactor-fuel-measurement facility desıgn

UNC

KING Reactor Mock-up

1967

BEING BUILT

LASL, Kiva I

\section{B. Military}

\begin{tabular}{llll}
\hline Facility & Subject of current expenment or study & Designation & Startup \\
\hline $\begin{array}{c}\text { OPERABLE } \\
\text { Bettis }\end{array}$ & Surface-ship physics ${ }^{77}$ & SS-CF & 1957
\end{tabular}




\section{B. Military (Continued)}

\begin{tabular}{llll}
\hline Facility & Subject of current experiment or study & Designation & Startup \\
\hline OPERABLE (Continued) & & \\
Bettis & High-temperature physics and mock-up & HTTF & 1959 \\
Bettis & Physics measurements 77 & CCFB & 1960 \\
Bettis & Clean critical experiments 7 CCX & 1961 \\
KAPL & Full Core Physics Experiment & FCPE & 1970 \\
KAPL & Flexible Critical Experiments & FPR & 1956 \\
KAPL & Cold Water Expenments & CWA & 1958 \\
KAPL & High-temperature high-pressure physics and mock-up & PTR & 1958 \\
KAPL & Cold Water Reactor test assembly & CWTA & 1960 \\
Lockheed & RER core configurations & CERF & 1958 \\
LASL, Kiva II & Critical-configuration safety tests & Comet II & 1953 \\
LASL, Kiva II & Plated bare-plutonium sphere studies & Jezebel & 1954 \\
LASL, Kiva II & Spherical metal cores in thick metal reflector & Flattop & 1957 \\
LASL, Kiva II & Water-1mmersion safety tests & Water tank & 1961 \\
RFP & Critical-configuration safety tests & NSF & 1965 \\
BEING BUILT & & & \\
LASL, Kiva II & U(10)-metal cylinder in thick metal reflector & Big ten & \\
\hline
\end{tabular}

\section{FOOTNOTES}

1 Power capacity figures are based on the best available information In all instances thermal capacity of the nuclear reactor is given, the electrical output, when shown, is the net electrical capacity of the power plant For reactors being built or planned, plant capacity is rounded to the nearest hundred kilowatts Where a plant has a stretch capacity, the initial capacity is given until the stretch value is approved

2 The Shippingport station is provided with a turbogenerator rated at $90,000 \mathrm{~kW}(\mathrm{e})$ net Use of a heat dissipation system permits operation at $150,000 \mathrm{~kW}(\mathrm{e})$ gross equivalent on core 2 Power operation with core 2 began Apr 30, 1965

3 This facility is regulated by the AEC Director of Regulation and has been issued an operating license (or authorization) or a construction permit, or an application for same has been submitted

4 This project is under the Power Demonstration Program
20 EOCR construction was terminated in December 1962 The facility was mothballed prior to operation

21 The EBWR achieved $100,000 \mathrm{~kW}(\mathrm{t})$ on Nov 11, 1962 Operatıon of EBWR in the Bollıng Water Program was closed out in December 1962 The reactor was used in support of the Plutonium Recycle Program and attaned criticality using plutonium as its principal fuel on Sept 22,1965 In support of that program it operated at power levels as high as $70,000 \mathrm{~kW}(\mathrm{t})$ Operation in that program was completed in June 1967

22 The MSRE was placed in standby in December 1969 pending determination of final disposition

23 SiOFS 4 operated in orbit during April-May 1965 Operation terminated unexpectedly after 43 days at power, probably owing to a sequence of fallures of electrical components of the spacecraft with resulting spurious commands 
5. In the Consolidated Edison Indian Point Station, the $615,000 \mathrm{~kW}(\mathrm{t})$ is increased by an oll-fired superheater to produce 265,000 net $\mathrm{kW}(\mathrm{e})$.

6. New York State Electric and Gas Corp. on Apr. 11, 1969, announced indefinite postponement of the Bell Station.

7. The Hallam Nuclear Power Facility was shut down in September 1964 due to moderator can falures. In August 1965 the Commission terminated its contract with Consumers Public Power District for operatıon of the nuclear plant. In May 1966 CPPD turned down their option to purchase the plant. In June 1966 the AEC announced deactivation and dismantling of the nuclear facility.

8. The last CVTR shutdown occurred Jan. 24, 1967. A license amendment issued June 14,1967 , authorizes CVNPA to possess but not operate the CVTR.

9. The Pathfinder Plant has been shut down since November 1967 On Sept. 9 1968, Northern States Power Company announced plans to install gas-fired boilers for operation the summer of 1969 .

10. The $58,200 \mathrm{~kW}(\mathrm{t})$ capacity of the Elk River Reactor is increased to $73,000 \mathrm{~kW}(\mathrm{t})$ by a fossil-fured superheater to produce 22,000 net $\mathrm{kW}(\mathrm{e})$. Thermal capacity of the reactor is equivalent to about $16,000 \mathrm{~kW}(\mathrm{e})$, the $14,800 \mathrm{~kW}(\mathrm{t})$ from the superheater is equivalent to about $6000 \mathrm{~kW}(\mathrm{e})$. Plant was shut down due to technical problems in February 1968, on Mar. 20, 1970, RCPA rejected an option to purchase ERR.

11. N Reactor, an AEC-owned reactor for production of special nuclear materials, also produces steam that is supplied to the adjacent electric generating plant, owned and operated by Washington Public Power Supply System (WPPSS). Initıal electric power generation began Apr. 8, 1966. Full gross power output of 800 MW(e) utılizing N Reactor steam was achieved on Dec. 9, 1966.

12 Midland Unit 1 supplies $3,625,000$ pounds per hour of process steam, and Unit 2 supplies 425,000 pounds per hour.

13. No footnote.

14. This facility was orignally built and operated in 1954 as the Bollng Reactor Experiment No. 2 (BORAX-2). With the addition of a turbogenerator, it operated during 1955 as BORAX-3 and on July 17, 1955, produced sufficient electricity to light and power Arco, Idaho-a U. S. first BORAX 4, a further modification, operated from December 1956 to June 1958 when the experıment was shu down.

15. OMRE demonstrated the technical and economic feasibility of using liquid hydrocarbon terphenyls as coolant and/or moderator.

16 The EBOR reactor experiment was terminated in December 1966 prior to the completion of construction.

17. In a trial run on Dec. 21 and 22, 1951, EBR-1 generated the world's first electric power from nuclear energy and was the first to demonstrate, in July 1953, the feasibility of breeding and the compatibility 'with breeding economy of sodium-potassium alloy as a liquid-metal coolant. It operated with a plutoniumbearıng core (Mark IV) from November 1962 to December 1963. The reactor was decommissioned and dismantled early in 1964. The facility was dedicated as a historic landmark Aug. 26, 1966.

18. SRE operated at $20 \mathrm{MW}(\mathrm{t})$ until shut down in February 1964 for modification to permit an increase in power level to $30 \mathrm{MW}(\mathrm{t})$. On Dec. 2, 1966, the AEC announced deactivation of SRE.

19. The EGCR project was terminated in January 1966 prior to the completion of construction. shutting down the reactor An 1dentical ground-test unit, S10FS-3, operated successfully for more than a year before being shut down in 1966. Another flight system unit, S10FS 5 , is in storage at Santa Susana, Calif.

24. Typical space propulsion rockets operate for 20 to $40 \mathrm{~min}$. Therefore space propulsion reactor experiments in context with other reactor applications operate for minutes rather than years

25 In August 1958 the MTR was operating up to $30,000 \mathrm{~kW}(\mathrm{t})$ using plutonium as fuel It demonstrated the ability of plutonium fuel elements to perform satisfactorily in a high flux power reactor. Operation as a test reactor was terminated on June 30,1969 , and a ${ }^{240} \mathrm{Pu}$ (Phoenix) core run in FY 1970. Facility in standby for possible use by others in FY 1972 .

26 This reactor is basically the same as the SNAP-10A Transient Test Reactor No. 1 (SNAPTRAN-1) that operated at NRTS from 1963-1965 It was moved from NRTS to its present location in the SNAP Environmental Test Facility. It is being used to evaluate the effects of separated ${ }^{155} \mathrm{Gd}$ as a burnable poison and as a shut-down agent in the event of water immersion.

27. The SNAPTRAN series of experiments was designed to develop, in a land-based environment, safety information on space auxiliary power reactors through excursion testing at various temperatures and rates of reactivity insertion. The destructive experiments approach the maximum credible accidents postulated for SNA.P reactor systems.

28 SPERT-II has been shut down and placed in standby for possible future use.

29. The BSR-2, which became operable in 1959 , is a stainless-steel $\mathrm{UO}_{2}$ core that can be used alternately in the same facility with BSR 1 (aluminum-alloy core)

30. The AE-6, also designated WBNS, was built and first operated at Downey, Calif. It was moved to Santa Susana in 1956.

31. This TRIGA reactor is capable of being pulsed and of steady state operation.

32. The RER was previously used in the terminated Aircraft Nuclear Propulsion Program. A license authorizing Lockheed to operate the reactor as a commercial facility was issued in July 1962, and in August 1962 the USAF transferred the facility to the General Services Administration. Lockheed acquired title to the facility in March 1965.

33 This TRIGA reactor is licensed for routine pulsing of 4 dollars and 60 cents in excess-reactivity insertions

34. This reactor was previously designated STF for SNAP Sheld Test Facility.

35. The HPRR was previously operated in the Nevada BREN facilty. It is now installed in the Dosımetry Applications Research Facility.

36 This reactor was operated in the USAEC Atoms for Peace Exhibit in Vienna, Austria, in June 1963, in Belgrade, Yugoslavia, in September 1963, in Madrid, Spain, in April 1964, in Lisbon, Portugal, in April 1965, in Utrecht, Netherlands, in March 1966, in Dublin, Ireland, in September-October 1966, Ankara, Turkey, in April-May 1967, Tehran, Iran, in November-December 1967, Taıpeı, Tarwan, in April-May 1968, Seoul, Korea, in September-October 1968, and is scheduled for Manila, Philippines, in February-March 1969, it is scheduled for Bucharest, Romania, in October 1969

37. The APFA-III was previously operated as the KUKLA Prompt Critıcal Assembly at Lawrence Radiation Laboratory at Livermore, Calıf.

38. Th1s reactor is scheduled to operate initially in São Paulo, Brazıl, in October 1969. 
39. In 1943 the Manhattan Engineer District disassembled Chicago Pile 1 and rebuilt it at Palos Park, Ill., as Chicago Pile 2 CP-2 had a thermal-power level of $10 \mathrm{~kW}$

40. This reactor was shipped abroad for exhibition purposes in the USAEC Atoms for Peace Exhibit in the Tokyo International Trade Fair in 1959, and in Cairo, Egypt, and Lahore, Pakistan, in 1960

41. This TRIGA-Mk II was operated at the New Delh1 World Agricultural Fair in 1960 It has been dismantled for storage in Californıa by GGA.

42. In 1965 and 1966 this reactor was operated at Sandia, N Mex as SNARE. Prio to that time it operated at the National Reactor Testing Station, Idaho, as the Shield Test Pool Reactor (Susie) in the Aircraft Nuclear Propulsion Program from 1959 to 1962 It was shut down in 1966 and transferred to Lousiana State University in June 1966.

43. The SPR was retıred in April 1967 and will be used as a laboratory device in a subcritical configuration only.

44. Until mid-1967, FRAN was operated by UCLRL at the Nevada Test Site It is now located in the former ML-1 reactor area at NRTS.

45 After the assembly and operation of this reactor in the government exhibit at Geneva in September 1958, it was dismantled and returned to ANL, where it was rebuilt as a $250-\mathrm{kW}(\mathrm{t})$ Juggernaut.

46. AGN-201-104 operated at the University of Akron (Ohio) from 1957 until transferred to the Georgia Institute of Technology in 1967.

47 AGN-201-112 was operated at the University of Calıfornia, Berkeley, beginning in 1957. The University of New Mexico filed an application in April 1966 for transfer and reconstruction of the reactor at a site on its campus The reactor achieved criticality at the University of New Mexico on Oct. 7, 1966.

48. The Puerto Rico Nuclear Center Pool Reactor is being converted to a $2 \mathrm{MW}$ TRIGA with startup scheduled in 1970

49 The University of Wisconsin reactor has been modified for 1000-kW steady stat operation with a TRIGA type core. Power level was $250 \mathrm{~kW}$ prior to modification in 1967

50. The Nuclear Science Center Reactor at Texas A\&M University has been modified for $1000-\mathrm{kW}$ steady-state operation with a TRIGA type core Power level was $100 \mathrm{~kW}$ prior to modification in 1968

51 In 1967 the original MTR-type core of the Washington State University reactor was replaced by a modified TRIGA-type core and control system, and the steady-state power level was increased from 100 to $1000 \mathrm{~kW}(\mathrm{t})$

52. From 1955 to 1965 the Penn State reactor was operated as a $200 \mathrm{~kW}(\mathrm{t})$ pool-type reactor fueled with MTR-type elements

53. In 1957-1962, AGN 201M-105 was owned and operated by the National Nava Medical Center, Bethesda, Md. Title to the reactor was transferred to New York University early in 1964 A license to operate was issued in April 1967.

54. The AGN-201P-103 was operated at San Ramon, Callf, by Aerofet-Genera Corporation from 1957 to 1966. In April 1967 Idaho State University applied for a license to operate the reactor at Pocatello, Idaho

55 The core of the Michigan State University reactor operated in the University of Illınors TRIGA facility from 1960 until transferred in 1968

56. This reactor was originally operated by North Carolina State University as the Raleigh Research Reactor (RRR). It was transferred in March 1966 to Mississippi
Lake, Panama Canal Zone, and began producing power to the Panama Canal power grid on Oct 5,1968

60. The PM-2A was shut down on July 9, 1963, and dismantled during April-June 1964 The reactor vessel was then used by the AEC at the National Reactor Testing Station, Idaho, for NDT (nil ductility transition temperature) investigations of materials that had been subjected to long term irradiation. Defects were sequentially introduced into the vessel wall during a series of tests involving pressure and temperature conditions which exceeded the range permitted in operating nuclear power plants. The final test on Nov, 18,1966, resulted in a brittle fracture under conditions even more severe than those which had been previously predicted to cause falure The test program confirmed laboratory data on the adequacy of reactor-operating limitations to prevent brittle fracture of a pressure vessel. The remaining parts of the primary system and the secondary system are in storage pending commitment by the Army for final disposition.

61. The abbreviations used here are defined as follows SSN, Submarıne (Nuclear Propulsion), SSBN, Fleet Ballıstic Missile Submarine (Nuclear Propulsıon), DLGN, Guided Missile Frigate (Nuclear Propulsion), CGN, Guided Missile Cruiser (Nuclear Propulsion), CVAN, Attack Aircraft Carrier (Nuclear Propulsion)

62 The USS SEAWOLF, originally commissioned with a sodium-cooled reactor in March 1957, was recommissioned with a pressurized-water reactor on Sept 30 , 1960.

63. The USS THRESHER (SSN593) was lost in the Atlantic on Apr. 10, 1963 The USS SCORPION (SSN 589) was lost in the Atlantic on May 21,1968

64. The Tory II-C was successfully tested at full desıgn power during May 1964 Subsequent to cancellation of the Pluto program on July 1, 1964, the reactor was placed in the Pluto disassembly building at NTS for storage.

65 In addition to the export power reactors listed, Westinghouse provided the design and furnished nuclear components, including fuel elements, control rods, and instrumentation for the $115-\mathrm{MW}(\mathrm{e})$ Belgium BR-3 pressurized-water reactor at Mol.

66. This $L 77$ reactor was operated in the commercial exhibit of the 1958 International Conference in Geneva and in the USAEC Atoms for Peace Exhibits in Beirut, Lebanon, in October 1961, in Athens, Greece, in May 1962, and in Bangkok, Thalland, in November 1962.

67 This is the 1955 Geneva Conference reactor rebuilt with increased power and now operating at Wue renlingen, Switzerland.

68 This $30 \mathrm{KW}(\mathrm{t})$ TRIGA Mk I is capable of power levels to $100 \mathrm{KW}$ for limited periods

69 This TRIGA reactor was operated at the 1958 International Conference in Geneva prior to shipment to the University of Lovanium. It began operating at the University of Lovanium in June 1959 It is the first reactor to be operated on the African continent.

70. This reactor was sold through GGA licensee, Gutehoff nungshuette Sterkrade A.E

71. The Netherlands research reactor was originally operated at the Amsterdam International Exhibition in June 1957, major portions of the exhibition reactor system were used to fabricate the present reactor. 
State University for reactivation. The original $10-\mathrm{kW}$ research reactor was started up in 1953 and was dismantled in 1955. The reactor was reactivated with a 500-watt core in March 1957. Early in 1959 the reactor was modified for 100-watt operation and moved to a new location in the Nuclear Science Laboratory, and a new $10-\mathrm{kW}$ heterogeneous-core Argonaut type reactor was built in the original RRR sheld vacated by the homogeneous core The RRR was dismantled by N C. State in 1963

57. Reactors in the Army Power Program are identtfied by symbolic nomenclature to reflect mobility characteristics, power range, development sequence, and field sequence. The first capital letter indicates mobility characteristics $S$ (stationary operation), not designed for subsequent relocation, $P$ (portable), semimobile, stationary operation, capable of being dismantled and reassembled for use in successive locations, and $M$ (mobile), capable of being moved intact, or virtually intact, for use in successive locations The second capital letter indicates the power range as measured by design capacity for contınuous operation L (low), 100 to $1000 \mathrm{~kW}(\mathrm{e}) \mathrm{M}$ (medium), 1000 to $10,000 \mathrm{~kW}(\mathrm{e})$, and $\mathrm{H}$ (high), $10,000 \mathrm{~kW}(\mathrm{e})$ or more. Arabic numerals indicate order in which plants having the same moblity and powe characterstics are ontated. If not followed by an same moblity and power characterstics are initated. additional letter, the designation indicates a prototype or pilot plant The las capital letter (when present) indicates the alphabetical order in which field plants of a specific type are initiated

58. The SM-1A produces 38 million Btu/hr for space heating in addition to electrical output

MH 1A was installed in the STURGIS (formerly the Liberty Ship CHARLES H. CUGLE), at Mobile, Ala Acceptance testing was performed at Fort Belvorr, Va, from April 1967 to June 27, 1967, when the Army accepted the plant from the Contractor In late July 1968 the plant was deployed to Gatun

72. This reactor was operated in the International Science Section of the Brussels Informational Exhibition, Apr. 15 to Oct. 1, 1958, pror to transfer to the University of Basel.

73. The AGN-201-111 was operated first in the USAEC Atoms for Peace Exhibit in Rome, Italy, in July 1958 and later in the commercial exhibit of the 1958 International Conference in Geneva prior to transfer to the University of Geneva

74. Prior to its sale to the University of Montevideo in 1966, this reactor was part of the USAEC Exhibit Program. It was in Buenos Aires, Argentina, in the fall of 1960, in Rio de Janeiro, Brazil, in the spring of 1961, in Lima, Peru, in the fall of 1961, in Mexico City in the spring of 1962, in Santiago, Chile, in the fall of 1962 in Bogotá, Colombia, in the spring of 1963, and in Montevideo, Uruguay, in the fall of 1963 The unit is expected to become operational in 1968 .

75. The SETF has capability for full-power SNAP tests and has been used for critical-assembly tests.

76. Zero-power experiments of historical interest previously conducted in ANL facility cells include the NAUTILUS core design (ZPR-1), the Savannah River reactor design (ZPR-2), and a series of fast-neutron studies (ZPR-4) and interactions between two basic systems (ZPR-5). The following experiments have been performed in the ZPR-7 facility thorium, uranium, deuterium criticals (THUD), and a series of flux-trap criticals for the Argonne High Flux Research Reactor.

77 The cell has one control panel for two pots. Experiments may be operated in either pot, but not simultaneously.

78 The B\&W Cells 2 and 3 share a control panel, only one cell can be operated at any one time.

79 The NASA ZPR-I and ZPR-II systems operate from the same control panel, but it is possible to operate only one system at a tıme. 


\section{NUCLEAR POWER PLANTS IN THE UNITED STATES}

The nuclear power plants included in this map are ones whose power is being transmitted or is scheduled to be transmitted over utility electric power grids and for which reactor suppliers have been selected

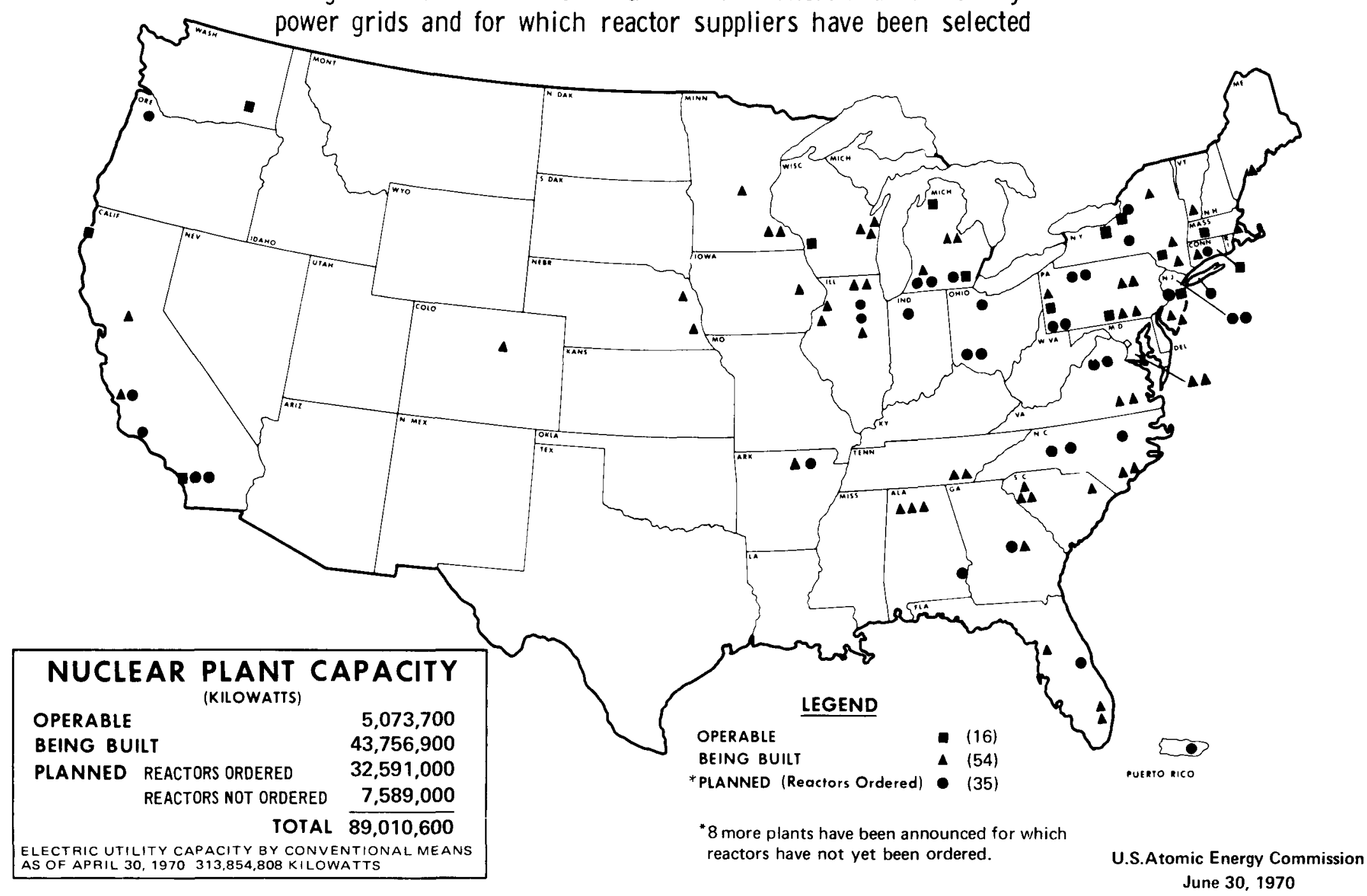


[REACTORS FOR EXPORT not included in the index (see pages 26 to 29).]

$$
\text { A1W, } 24
$$

Aberdeen Pulsed Reactor Facility [APRF], 25

Accelerator Pulsed Fast Critical Assembly [APF A III] , 18, 31

Advanced Test Reactor [ATR ], 15

AE 6 (WBNS), 17

Aerojet-General Corp., AGN-201P 103,

$$
18
$$

Aerospace Systems Test Reactor

$$
\text { [ASTR ], } 24
$$

\section{AFRRI, 25}

AFSR, 17

AGN Industrial Reactor [ AGNIR ], 18

AGNIR, 18

Aguirre Nuclear Power Plant, 11

Arrcraft Reactor Experiment [ARE] , 24

Alabama Power Company, 10

ALRR, 16

American Radiator \& Standard Sanitary

$$
\text { Corp. [UTR 1], } 18
$$

Ames Laboratory Research Reactor

$$
\text { [ALRR], } 16
$$

AMRR, 25

Annular Core Pulsed Reactor ITRIGA ACPR ], 18

APFA-III, 18, 31

APRF, 25

ARE, 24

Argonaut [CP 11], 17

Argonne $\mathrm{CP} 3$, rebult as $\mathrm{CP}-3^{\prime}$ [ $\mathrm{CP}^{\prime}$ ], 18

Argonne Low Power Research Reactor [Juggernaut ], 19

Argonne National Laboratory, AGN

$$
\text { 201-108, } 17
$$

Argonne Nuclear Assembly for Unıversity Training [Argonaut (CP 1 1)], 17

Argonne Research Reactor [CP 5], 16 Argonne Thermal Source Reactor

$$
\text { [ATSR], } 17
$$

Arkansas Nuclear One, 9

Unit 2, 11
Armed Forces Radıobıology Research Institute [AFRRI], 25

ARMF I, 29

ARMF II, 29

Army Materials Research Reactor

[AMRR], 25

ARR(L 54), 18

ASTR, 24

Atomics International L 47, 18

ATR, 15

ATRC, 29, 30

ATSR, 17

\section{B Reactor, 2}

Babcock \& Wilcox Lynchburg Pool

$$
\text { Reactor [LPR ], } 17
$$

Babcock \& Wilcox Nuclear Development Center Test Reactor [BAWTR], 16

Bally Generating Station, 1

Battelle Memorial Institute [BRR], 17

BAWTR, 16

Beaver Valley Power Station Unit 1,9

Bell Station, 11

BGRR, 16

Big Rock Point Nuclear Plant, 7

Big ten, 32

Bılogical Research Reactor [JANUS] 18

Bollıng Nuclear Superheater Power Station, 11

Bolling Reactor Experiment

No 1 [BORAX-1], 12

No 5 [BORAX 5], 13

Bollng Reactor Experments [ BORAX

$$
2,3,4], 13
$$

BORAX-1, 12

BORAX-2,3,4, 13

BORAX $-5,13$

Brigham Young University, L-77, 20

Brookhaven High Flux Beam Research Reactor [HFBR], 16

Brookhaven Medical Research Redctor

$$
\text { [MRR ], } 16
$$

\section{REACTOR INDEX}

Brookhaven Neutron Source Reactor

No, 2 [PHRENIC], 19

Brookhaven Research Reactor [BGRR], 16

Browns Ferry Nuclear Power Plant

Unit 1,8

Unit 2, 9

Unit 3,9

Brunswick Steam Electric Plant

Unit 1,10

Unit 2,10

BRR, 17

BSR, 17

Bulk Shielding Redctor, 17

C Reactor

Graphite, 21

Heavy water, 21

Calvert Chffs Nuclear Power Plant

Unit 1, 9

Unit 2, 9

Carolina Power \& Light Co , 11

Carolinas Virgınıa Tube Reactor, 11

Cathohic University of America, AGN201101,19

CCFA, 30

CCFB, 32

$\mathrm{CCX}, 32$

CERF, 32

Chicago P1le 1 , rebuilt as CP 2 [CP 2], 18

Clementine, 18

Colorddo State University

AGN 201-109, 19

TRIGA Mk III, 20

Columbia University, TRIGA Mk II, 20

Comet II, 32

Consolidated Edison Co , 1

Cooper Nuclear Station, 9

Cornell University

TRIGA-Mk II, 20

Zero Power Reactor [ZPR], 20
CP-2, 18

CP-3, 18

CP 5,16

Crystal River Plant, Unit 3, 9

Curtiss Wright Nuclear Research Labora tory of the Commonwealth of Pennsylvan 1 a, 18

CWA, 32

CWTA, 32

D Reactor, 21

DIG, 24

Davis Besse Nuclear Power Station, 10

Destroyer Reactor Prototype [D1G] 24

Drablo Canyon Nucledr Power Plant

Unit 1,9

Unit 2,10

Diamond Ordnance Radidtion Facility [DORF], 25

Donald C Cook Nuclear Plant

Unit 1,9

Unit 2,9

DORF 25

Dow Chemical Co, TRIGA Mk I, 18

DR Reactor, 21

Dresden Nuclear Power Station

Unit 1, 7

Unit 2, 8

Unit 3,8

Duane Arnold Energy Center, Unit 1, 10

Duke Power Co , 2 units, 11

EBOR, 13

EBR 1, 13

EBR 2, 12

EBWR, 13

ECEL, 30

Edwin I Hatch Nuclear Plant

Unit 1, 9

Unit 2, 11

EGCR, 13

Elk River Reactor, 1 
Engineering Test Reactor [ETR], 15

Enrico Fermi Atomic Power Plant

Unit 1, 7

Unit 2, 10

EOCR, 13

ESADA Vallecitos Experimental Superheat Reactor [EVESR], 13

ETR, 15

ETRC, 29

EVESR, 13

Experımental Beryllium Oxıde Reactor [EBOR], 13

Experimental Bolling Water Reactor [EBWR], 13

Experımental Breeder Reactor

No 1 [EBR 1], 13

No 2 [EBR-2], 12

Experimental Gas Cooled Reactor

$$
\text { [EGCR], } 13
$$

Experimental Organic Cooled Reactor [EOCR], 13

Experimental Propulsion Test Reactor

[TORY II A], 24

[TORY II C], 24

F Reactor, 21

Fast Burst Reactor Facılity [FBRF], 25

Fast Flux Test Facility [FFTF], 15

Fast Source Reactor [AFSR], 17

FBRF, 25

FCPE, 32

FFTF, 15

Flattop, 32

Forked River Nuclear Generating Station

Unit 1, 11

Fort Calhoun Station, Unit 1, 9

Fort St. Vrain Nuclear Generating Station, 9

FPR, 32

FRAN, 19

Fuel Element Test Bed NF-1, 14

Fuel Element Test Reactor

[Pewee-1], 15

[Pewee-3], 14

[Pewee-4], 14

Gas Cooled Reactor Experiment [GCRE], 24

GCRE, 24

General Electric Nuclear Test Reacto [NTR], 17

General Electric Test Reactor [GETR], 15

gia Institute of Technology, AGN-

201104,19
Idaho State University, AGN 201P 103

$$
20
$$

Illnois Institute of Technology Research

Institute [ARR(L-54)], 18

Indian Point Station

Unit 1,7

Unit 2,8

Unit 3, 9

Industrial Reactor Laboratories, Inc., 16

Intrinsic Subcritical Assembly,

SNAPTRAN-1, 16

Iowa State University, UTR-10,19

James A. FitzPatrick Nuclear Power Plant, 10

JANUS, 18

Jezebel, 32

Joseph M. Farley Nuclear Plant, 10

Juggernaut, 19

K Reactor, 21

Kansas State University, TRIGA Mk II,$$
20
$$

KEWB 17

KE Reactor, 21

Kewaunee Nuclear Power Plant, 9

Kinetic Experiment on Water Boilers [KEWB], 17

Kinglet, 31

Kiwl-A, 14

Kiw1-A Prime, 14

Kiw1 A3, 14

Kiw1-B 1 A, 14

KiW1-B 1 B, 14

Kiwl-B4A, 15

Kiw1-B4D, 15

Kiw1-B4E, 15

Kiw1-TNT, 17

Kiw1-Transient Test Reactor [ Kiw

TNT], 17

KW Reactor, 21

L Reactor, 21

La Crosse Bollıng Water Reactor, 8

LAMPRE-1, 13

LAPRE-1, 13

LAPRE 2, 13

Large Ship Reactor Prototy pe [AIW], 24

LaSalle County Nuclear Station

Unit 1, 11

Unit 2, 11

Leland Stanford University, 19
MRR, 16

MSRE, 13

MTR, 15

MUR, 17

N Reactor, 12

NASA Mock up Reactor [MUR], 17

NASA TR, 15

National Bureau of Standards [NBSR]

Natural Circulation Test Plant [S5G], 24

Naval Research Reactor [NRR], 25

NBSR, 16

Newbold Island Nuclear Generatıng Station

Unit 1,10

Unit 2, 11

New York Hall of Science, TRIGA Mk II, 20

New York University

AGN-201M 105, 20

TRIGA-Mk I, 20

NF-1, 14

Nine Mile Point Nuclear Station, 8

North American Rockwell Corp.

L 77, 17

Water Boller Neutron Source [AE6(WBNS ], 17

North Anna Power Station

Unit 1,10

Unit 2, 10

North Carolina State University

Graphite/water, 19

PULSTAR, 20

Northrop Corporate Laboratories, TRIGA-Mk F, 17

NRR, 25

NRX A2, 15

NRX-A3, 15

NRX-A4/EST, 15

NRX-A5, 15

NRX-A6, 15

NSCR, 19

NSF, 32

NTR, 17

Nuclear Effects Reactor

[FRAN], 19

[Super KUKLA], 25

Nuclear Engineering Test Reactor [NETR ], 25

Nuclear Rocket Engine Reactor Experıment (NERVA)

[NRX A2 ], 15

[NRX A3], 15
Peach Bottom Atomic Power Station

Unit 1,

Unit 2, 8

Unit 3,9

Penn State TRIGA Reactor (Pennsyl vania State University) [PSTR ], 20

Pennsylvania Power \& Light Co.

Unit 1, 11

Unit 2,1 1

Pewee-1, 15

Pewee-2, 14

Pewee-3, 14

Pewee-4, 14

Philadelphia Electric Company

Unit 1,10

Unit 2, 11

Phoebus 1A, 15

Phoebus 1B, 15

Phoebus 2A, 15

PHRENIC, 19

Physical Constants Test Reacto [PCTR], 17

Pilgrim Station, 8

Pıqua Nuclear Power Facility, 11

Plum Brook Reactor Facility [NASATR ], 15

Plutonium Recycle Test Reactor [PRTR], 13

PM-1, 22

PM-2A, 22

PM-3A, 22

Point Beach Nuclear Plant

Unit 1,8

Unit 2, 8

Portable Medium Power Plant

No. 1 [PM-1], 22

No. 2A [PM-2A], 22

No 3A [PM-3A], 21

Power Burst Facility [PBF], 16

Prairie Island Nučlear Generatıng Plan Unit 1,9

Unit 2,10

Process Development Pile [PDP], 21

PRR, 17

PRTR, 13

PSTR, 20

PTF, 31

Public Service Electric \& Gas Co. 2 units, 10,11

Puerto Rico Nuclear Center

L-77, 19

Pool, 19

Purdue University, 20 
gia Tech Research Reacto GTRR ], 20

GETR, 15

Godiva-IV, 31

Ground Experımental Engine Exper1ment

XE-Prime, 15

Ground Test Reactor, [GTR ], 25

GTR, 25

GTRR, 20

Gulf General Atomic Incorporated

Prototype Reactor, TRIGA-Mk I, 17

Prototype Reactor, TRIGA-Mk III, 18

Prototype Reactor, Advanced TRIGA-

Mk F, 17

Exhıb1t Reactor, TRIGA-Mk II, 18

Critical Assembly, 29, 31

H. B. Robinson S.E. Plant, Unit 2,

H Reactor, 21

Haddam Neck Plant, 8

Hallam Nuclear Power Facility Sheldon Station, 11

Hanford 305 Test Reactor [HTR], 21

Health Physics Research Reactor [HPRR], 17

Heat Transfer Reactor Experıment

No 1 [HTRE-1], 24

No. 2 [HTRE-2], 24

No. 3 [HTRE-3], 24

Heavy Water Components Test Reactor [HWCTR], 13

HFBR, 16

HFIR, 16

High Flux Isotope Reactor [HFIR], 16 High Temperature Lattice Test Reactor [HTLTR], 18

Homogeneous Reactor Experıment

No. 1 [HRE-1], 12

No. 2 [HRE-2[, 13

Honey comb, 31

HPR R, 17

HRE-1, 12

HRE-2, 13

HTLTR, 18

HTR, 21

HTRE 1,24

HTRE-2, 24

HTRE-3, 24

HTTF, 32

Humboldt Bay Power Plant, Unit 3, 7

Hutchinson Island, Unit 1, 10

HWCTR, 13

HYDRO, 17

HYPO, 18
Unit 2, 1

LITR, 19

Livermore Pool Type Reactor [ LPTR]$$
17
$$

Livermore Water Bo1ler [LIWB], 18

LIWB, 18

Lockheed Aurcraft Corp., 18

LOFT, 16

Los Alamos Fast Reactor [Clementine], 18

Los Alamos HYDRO Reactor [HYDRO], 17

Los Alamos Molten Plutonium Reactor Experiment [LAMPRE 1], 13

Los Alamos Power Reactor Experiment No. 1 [LAPRE-1] , 13

No. 2 [LAPRE-2], 13

Los Alamos Water Boller

[HYPO], 18

[SUPO], 17

Loss of Fluid Test [LOFT], 16

Loussiana State University Nuclear

Science Center [SNARE], 18

Low Intensity Test Reactor [LITR ], 19

Lowell Technological Instıtute, 20

LPR, 17

LPT, 17

LWBCC, 30

Maine Yankee Atomic Power Plant, 9

Malibu Nuclear Plant, Unit 1, 11

Manhattan College, 20

Massachusetts Institute of Technology

[MITR], 19

Materials Testing Reactor [MTR], 13

MH-1 A, 22

Michıgan State Unıversity, TRIGA-Mk I, 20

Midland Nuclear Power Plan

Unit 1,12

Unit 2,12

Millstone Nuclear Power Station

Unit 1,8

Unit 2,10

MITR, 19

Mississippi State Unıversity [RRR], 20

ML 1, 24

Mobile Low Power Plant No 1 [ML-1] 24

Molten Salt Reactor Experiment

[MSRE] , 13

Monticello Nuclear Generatıng Plant, 8
$[N R X$ A5], 15

[NRX-A6], 15

Nuclear Rocket Reactor Engine System Test (NERVA) [NRX-A4/EST], 15

Nuclear Rocket Reactor Experıment

[Kiwi-A], 14

[ Kiwi-A Prime], 14

[Kıwı A3 ], 14

[Kiwı B1 A], 14

[Kıw1-B1B], 14

[Kıw1-B4A], 15

[Kiwa-B4D], 15

[Kiwl-B4E], 15

[Phoebus 1A], 15

[Phoebus 1B], 15

[Phoebus 2A], 15

Nuclear Science Center Reactor, Texas A\&M University $[\mathrm{NSCR}], 19$

Oak Ridge Graphite Reactor [X 10], 18

Oak Ridge Research Reactor [ORR], 16

Oconee Nuclear Station

Unit 1, 8

Unit 2,9

Unit 3,9

Oh1o State University, 19

Oklahoma State University of Agriculture and Applied Science, AGN 201102,19

Omaha Veterans Administration Hospital, TRIGA Mk I, 17

Omega West Reactor [OWR], 16

OMRE, 13

Oregon State University

AGN 201-1 14, 19

TRIGA-Mk II, 20

Organic Moderated Reactor Experiment [OMRE], 13

ORR, 16

OWR, 16

Oyster Creek Nuclear Power Plant,

Unit 1, 8

P Reactor, 21

Palisades Nuclear Power Station,

Unit 1,8

Pathfınder Atomic Power Plant, 11

Pawling Research Reactor [PRR], 17

PBF, 16

PCA, 31

PCTR 17

PDP, 21
Quad-Cities Station

Unit 1,8

Unit 2, 8

R Reactor, 21

Radıation Effects Reactor [RER], 17

Rancho Seco Nuclear Generatıng

Station, Unit 1, 9

Reed College, TRIGA-Mk I, 20

RER, 17

Resonance Test Reactor [RTR ], 21

Rhode Island Nuclear Science Center, 18

Robert Emmett Ginna Nuclear Power Plant, Unit 1, 8

RTR, 21

S1C, 24

S1G, 24

S1W Reactor Facility [S1W], 24

S2DS, 14

S3G, 24

S5G, 24

S8DR, 14

S8ER, 14

S1OFS 1, 14

S10F S-3, 14

S1 OFS $-4,14$

S10FS-5, 14

Salem Nuclear Generating Station

Unit 1,9

Unit 2, 9

Sandıa Engineerıng Reactor [SER], 16

Sandia Pulsed Reactor

[SPR ], 18

[SPR-II], 18

San Onofre Nuclear Generatıng Station

Unit 1,7

Unit 2, 11

Unit 3,11

Savannah River Test Ple

[SR-305], 21

Saxton Nuclear Experimental Reactor Project, 12

$\mathrm{SCA}-5,30$

SCA-4A and 4B, 30

SCHIZO, 19

SEFOR, 12

Sequoyah Nuclear Power Plant

Unit 1,10

Unit 2,10

SER, 14,16

Shield Test and Irradiation Reactor, [STIR], 17 
Shıppıngport Atomıc Power Station, 7 Ships

Merchant, 12

Naval, 22, 23, 24

Shoreham Nuclear Power Station, 11

SL-1, 24

$\mathrm{SM}-1,22$

SM-1A, 22

Small Submarıne Reactor Prototype,

$$
[\mathrm{S} 1 \mathrm{C}], 24
$$

SNAP-2 Developmental System [S2DS] 14

SNAP-2 Experımental Reactor [SER], 14

SNAP-2/10A-TSF Shielding Exper1ment [SNAP TSF], 13

SNAP-8 Developmental Reactor.

[S8DR], 14

SNAP-8 Experımental Reactor [S8ER] 14

SNAP-10A Fight System

[S10FS-4], 14

[S1 0FS-5], 14

SNAP-10A Flight System Ground Test

-1], 14

No 3 [S10FS 3], 14

SNAP-10A Transient rest

No. 2 [SNAPTRAN 2], 17

No. 3 [SNAPTRAN-3], 16

SNAPTRAN 1,16

SNAPTRAN 2, 17

SNAPTRAN-3, 16

SNAP-TSF, 13

SNARE, 18

Sodtum Reactor Experıment [SRE

$$
\text { PEP], } 13
$$

Southwest Experımental Fast Oxıde Reactor [SEFOR], 12

\section{SP, 2}

Specral Power Excursion Reactor Test No. 1 [SPERT-1], 17

No. 2 [SPERT-2], 17

NO. 3 [SPERT-3], 17

No 4 [SPERT.

SPERT-1, 17

SPERT-2, 17

SPERT 3, 17

SPERT 4,16

SPR, 18

SPR II, 18

SR 305, 21

SRE PEP, 13

SS-CF, 31

Standard Pile [SP], 2

Stationary Low Power Plant No 1 [SL-1], 24

Statıonary Medıum Power Plant

No. 1 [SM 1], 22

No, 1 A [SM 1 A], 22

STIR, 17

STURGIS Floatıng Nuclear Power Plant

[MH-1 A] , 22

Submarme Advanced Reactor Prototype [S3G], 24

Submarine Intermediate Reactor Mark A [S1G], 24

Submarınes, 22, 23

Super KUKLA, 25

SUPO, 17

Surry Power Station

Unit 1,8

Unit 2,9

Test Bed Reactor [Pewee-2], 14

Tennessee Valley Authority, 10

Texas A\&M University

AGN-201-106, 19

NSCR, 19

Thermal Test Reactor

No, 1 [TTR-1], 25

No, 2 [TTR 2], 17

Three Mile Island Nuclear Station

Unit 1,9

Unit 2,9

TORY II A and TORY II-C, 24

Tower Shelding Reactor

No, 1 [TSR-1], 25

No. 2 [TSR 2], 25

TREAT, 16

Transient Reactor Test [TREAT], 16

Trojan Nuclear Plant, Unit 1, 10

TSR-1 and TSR-2, 25

TTR-1, 25

TTR-2, 17
Turkey Point Station

Unit 3,8

Unit 4,9

UCNR, 16

UFTR, 19

UHTREX, 13

Ultra High Temperature Reactor Exper1ment [UHTREX], 13

UMNE 1,19

Union Carbide Corp. [UCNR ], 16

USAEC European-Asian Exhibit Program, 18

USAEC Latın American Demonstratıon Reactor Center, 18

U. S Geological Survey Laboratory, TRIGA-Mk I, 18

U S. Naval Postgraduate School, AGN 201100,25

University of Arızona, TRIGA Mk I, 19 University of Calıfornı, TRIGA Mk I and TRIGA-Mk III, 20

University of Calıfornı dt Los Angeles, School of Engineering and Apphed Scrence, Educator, 19

University of Delaware, AGN-201-113, 19

University of Flonda [UFTR], 19

University of Illınois, TRIGA Mk III, 19

$$
\text { LOPRA, } 20
$$

(4180,20

University of Maryland [UMNE 1], 19

University of Michigan (Ford Nuclear Reactor), 19

University of Missourı, 20

University of Missourı at Rolla, 20

University of Nevada, L-77, 20

University of New Mexico, AGN

$$
\text { 201M-112, } 19
$$

Unversity of Oklahoma, AGN-211 102, 19

University of Texas, TRIGA-Mk I, 20

University of Utah, AGN-201-107, 19

University of Virginia, 19

University of Washington, Educator, 20

University of Wisconsin, 19

University of Wyoming, L 77, 19

UTR Test Reactor, 18
UTR 1,18

Vallecitos Bollıng Water Reactor |VBWR ], 13

VBWR, 13

Vermont Yankee Generating Station, 8

Virginia Polytechnic Institute, UTR 10,

Walter Reed Resedrch Reactor [WRRR], 25

Washington State University, 20

Water tank, 32

West Virginia University, AGN 211-103,

Western New York Nuclear Research

Center, Inc. (State University of New

York), PULSTAR, 20

Westınghouse Testıng Reactor [WTR ] 15

William B. McGuire Nuclear Station

Unit 1,11

Unit 2,11

Willam H Zimmer Nuclear Power Station

Unit 1,10

Unit 2, 11

William Marsh Rice University, AGN-

211101,20

Worcester Polytechnic Institute, 19

WRRR, 25

WTR, 15

$X 10,18$

Xe-Prıme, 15

Yankee Nuclear Power Station, 7

ZEPO, 31

ZPPR, 30

ZPR, 20

ZPR 3, 30

ZPR 5, 30

ZPR-6, 30

ZPR-7, 30

ZPR-9, 30

Zion Station

Unit 1,8

Unit 2,9 